\begin{tabular}{|l|l|}
\hline & $\begin{array}{l}\text { HHS PUbic ACCOSS } \\
\text { Author manuscript } \\
\text { Nature. Author manuscript; available in PMC } 2016 \text { November } 04 .\end{array}$ \\
\hline
\end{tabular}

Published in final edited form as:

Nature. ; 533(7604): 481-486. doi:10.1038/nature17998.

\title{
NMDAR inhibition-independent antidepressant actions of ketamine metabolites
}

\author{
Panos Zanos $^{\mathrm{a}}$, Ruin Moaddel ${ }^{\mathrm{h}}$, Patrick J. Morris', Polymnia Georgiou ${ }^{\mathrm{a}}$, Jonathan Fischell ${ }^{\mathrm{d}}$, \\ Greg I. Elmer ${ }^{a, c, g}$, Manickavasagom Alkondon ${ }^{b}$, Peixiong Yuan', Heather J. Pribut ${ }^{a}$, \\ Nagendra S. Singh ${ }^{\mathrm{h}}$, Katina S.S. Dossou ${ }^{\mathrm{h}}$, Yuhong Fang ${ }^{\mathrm{i}}$, Xi-Ping Huangk ${ }^{\mathrm{k}}$, Cheryl L. Mayog, \\ Irving W. Wainer ${ }^{h, m}$, Edson X. Albuquerque ${ }^{b, c, f}$, Scott M. Thompson ${ }^{a, d}$, Craig J. Thomas $^{i}$, \\ Carlos A. Zarate Jr.j, and Todd D. Gould ${ }^{\mathrm{a}, \mathrm{c}, \mathrm{e}}$ \\ aDepartment of Psychiatry, University of Maryland School of Medicine, Baltimore, MD, USA. \\ bepartment of Epidemiology and Public Health, Division of Translational Toxicology, University of \\ Maryland School of Medicine, Baltimore, MD, USA. \\ 'Department of Pharmacology, University of Maryland School of Medicine, Baltimore, MD, USA. \\ dDepartment of Physiology, University of Maryland School of Medicine, Baltimore, MD, USA. \\ eDepartment of Anatomy and Neurobiology, University of Maryland School of Medicine, Baltimore, \\ MD, USA. \\ fDepartment of Medicine, University of Maryland School of Medicine, Baltimore, MD, USA. \\ 9Maryland Psychiatric Research Center, University of Maryland School of Medicine, Baltimore, \\ MD, USA.
}

hBiomedical Research Center, National Institute on Aging, National Institutes of Health, Baltimore, MD, USA.

Reprints and permissions information is available at www.nature.com/reprints.Users may view, print, copy, and download text and data-mine the content in such documents, for the purposes of academic research, subject always to the full Conditions of use:http:// www.nature.com/authors/editorial_policies/license.html\#terms

Correspondence and requests for materials should be addressed to TDG (gouldlab@me.com).

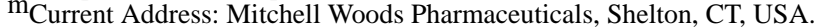

Supplementary Information is linked to the online version of the paper at www.nature.com/nature.

Author Contributions: PZ, RM, PJM, IWW, CJT, CAZ, and TDG were responsible for the overall experimental design. PJM, YF, and CJT synthesised the ketamine metabolites and deuterated ketamine derivatives, and provided mass spectrometer confirmations. Bioanalytical quantitation of ketamine and metabolites were performed by RM, NSS, and KSSD. PZ, PG, and HJP conducted and analysed the results of the behavioural and EEG experiments. XPH supervised and analysed the results of the binding experiments. PY performed the western blot experiments. EXA, MA, JF, and SMT helped design and analyze the electrophysiology experiments, which were conducted by MA, JF, and SMT. GIE and CLM conducted and analysed the results of the i.v. self-administration. PZ and TDG outlined and wrote the paper, which was reviewed by all authors.

The authors declare competing financial interests: IWW, RM, and CAZ are listed as co-inventors on a patent for the use of (2R,6R)hydroxynorketamine, (S)-dehydronorketamine and other stereoisomeric dehydro- and hydroxylated metabolites of (R,S)-ketamine metabolites in the treatment of depression and neuropathic pain. IWW, CAZ, RM, TG, PZ, CT, and PM are listed as co-inventors on a patent application for the use of $(2 \mathrm{R}, 6 \mathrm{R})$-hydroxynorketamine and $(2 \mathrm{~S}, 6 \mathrm{~S})$-hydroxynorketamine in the treatment of depression, anxiety, anhedonia, suicidal ideation and post-traumatic stress disorders. IWW, CAZ, RM, CT, and PM have assigned their patent rights to the U.S. government but will share a percentage of any royalties that may be received by the government. TG and PZ have assigned their patent rights to the University of Maryland Baltimore but will share a percentage of any royalties that may be received by the University of Maryland Baltimore. All other authors declare no competing interests. 
'Division of Preclinical Innovation, National Center for Advancing Translational Sciences, National Institutes of Health, Rockville, MD, USA.

jExperimental Therapeutics and Pathophysiology Branch, Intramural Research Program, National Institute of Mental Health, National Institutes of Health, Bethesda, MD, USA.

kNIMH Psychoactive Drug Screening Program, Department of Pharmacology and Division of Chemical Biology and Medicinal Chemistry, University of North Carolina Chapel Hill Medical School, Chapel Hill, North Carolina, USA.

\section{Abstract}

Major depressive disorder afflicts $\sim 16$ percent of the world population at some point in their lives. Despite a number of available monoaminergic-based antidepressants, most patients require many weeks, if not months, to respond to these treatments, and many patients never attain sustained remission of their symptoms. The non-competitive glutamatergic $N$-methyl- $D$-aspartate receptor (NMDAR) antagonist, (R,S)-ketamine (ketamine), exerts rapid and sustained antidepressant effects following a single dose in depressed patients. Here we show that the metabolism of ketamine to $(2 \mathrm{~S}, 6 \mathrm{~S} ; 2 \mathrm{R}, 6 \mathrm{R})$-hydroxynorketamine $(\mathrm{HNK})$ is essential for its antidepressant effects, and that the (2R,6R)-HNK enantiomer exerts behavioural, electroencephalographic, electrophysiological and cellular antidepressant actions in vivo. Notably, we demonstrate that these antidepressant actions are NMDAR inhibition-independent but they involve early and sustained a-amino-3-hydroxy-5methyl-4-isoxazole propionic acid (AMPA) receptor activation. We also establish that (2R,6R)HNK lacks ketamine-related side-effects. Our results indicate a novel mechanism underlying ketamine's unique antidepressant properties, which involves the required activity of a distinct metabolite and is independent of NMDAR inhibition. These findings have relevance for the development of next generation, rapid-acting antidepressants.

\section{Introduction}

Severe depression afflicts approximately 16 percent of the world population at some point in their lives and is associated with serious health and socioeconomic consequences ${ }^{1,2}$. Current pharmacotherapies, typically including monoaminergic-acting antidepressants, require prolonged administration (weeks if not months) for clinical improvement. This lag time, as well as a high non-response rate, emphasizes the need for better antidepressant medications ${ }^{3}$. The non-competitive glutamatergic $N$-methyl- $D$-aspartate receptor (NMDAR) antagonist, (R,S)-ketamine (ketamine), has demonstrated rapid and robust efficacy as an antidepressant by improving core depressive symptoms including depressed mood, anhedonia, and suicidal thoughts in treatment-refractory unipolar and bipolar depressed patients when administered at sub-anesthetic doses ${ }^{4-8}$. Remarkably, these actions are observed within hours following a single administration, and persist for approximately one week. While discovery of the ketamine's clinical antidepressant efficacy for the treatment of depression has elicited tremendous excitement in the field, its potential for widespread clinical use is limited due to its abuse liability and capacity to produce dissociative effects even when administered at low doses ${ }^{9}$. There are also unanswered questions regarding how ketamine works as an antidepressant, which is typically assumed to depend on direct NMDAR inhibition. However, the results of human treatment trials indicate that alternate 
NMDAR antagonists lack the robust, rapid, or sustained antidepressant properties of ketamine ${ }^{10}$.

\section{Role of NMDAR inhibition in ketamine action}

We compared the antidepressant-like effects of ketamine and the classical tricyclic antidepressant desipramine in the mouse forced-swim test (FST) at 1 hour (acute) and 24 hours (sustained) after administration (Fig. 1a). A $10 \mathrm{mg} / \mathrm{kg}$ dose of ketamine resulted in acute and long-lasting dose-dependent antidepressant effects in the FST, whereas the monoaminergic-acting antidepressant desipramine only decreased immobility time 1 hour post-injection. To date, most studies assessing ketamine's antidepressant effects are based on a commonly accepted view that ketamine and its $\mathrm{N}$-demethylated metabolite $(\mathrm{R}, \mathrm{S})$ norketamine are the active agents, whose clinical effects are due to inhibition of the NMDAR. Additional metabolites (Extended Data Figs. 1 and 2a,b) are considered clinically inactive since they do not induce anaesthesia ${ }^{11}$. To elucidate whether NMDAR inhibition is the main mechanism underlying the antidepressant effects of ketamine, we assessed the effects of ketamine's enantiomers (S)- and (R)-ketamine in the FST (Fig. 1b), noveltysuppressed feeding (NSF; Fig. 1c) and learned helplessness (LH; Fig. 1d) tests. While the NMDAR hypothesis of ketamine action would predict greater efficacy of (S)-ketamine since it is a 3-4 fold more potent inhibitor of the NMDAR than (R)-ketamine ${ }^{12,13}$, our results, in accordance with a recent report ${ }^{14}$, demonstrate a greater potency of $(\mathrm{R})$-ketamine in all three antidepressant-predictive tasks. Notably, this antidepressant effect does not result from higher brain levels of (R)-ketamine compared to (S)-ketamine (Extended Data Fig. 2c-e). Moreover, in contrast to ketamine, we demonstrated that the NMDAR antagonist MK-801, which binds at the same receptor site as ketamine, does not exert sustained (24-hour) antidepressant-like effects in the FST (Fig. 1e; also see ${ }^{15,16}$ ), or reverse social interaction deficits induced by chronic social defeat stress (Fig. 1g; Extended Data Fig. 3). These findings indicate a likely NMDAR inhibition-independent mechanism underlying the antidepressant responses of ketamine.

\section{Antidepressant actions of HNK}

Ketamine is stereoselectively metabolised into a broad array of metabolites, including norketamine, hydroxyketamines, dehydronorketamine, and the hydroxynorketamines (HNKs) ${ }^{17,18}$ (Fig. 2a; Extended Data Fig. 1). Following ketamine administration, (2S,6S; 2R,6R)-HNK is the major HNK metabolite found in the plasma and brain of mice (Extended Data Fig. 2a, b), and plasma of humans ${ }^{19}$. Similar to previous evidence revealing enhanced ketamine antidepressant responses in female rodents compared to males ${ }^{20,21}$, we observed greater antidepressant potency of ketamine in female mice (Fig. 2b), which was not associated with sex differences in ketamine-induced hyperlocomotion (likely mediated by NMDAR inhibition ${ }^{22}$; Extended Data Fig. 4a,b). In order to investigate whether these sexdependent antidepressant differences are explained by a different pharmacokinetic profile of ketamine in males versus females, we measured the levels of ketamine and its metabolites in the brains of mice following ketamine administration. While equivalent levels of ketamine and norketamine were found, $(2 \mathrm{~S}, 6 \mathrm{~S} ; 2 \mathrm{R}, 6 \mathrm{R})$-HNK was approximately three-fold higher in the brain of female mice compared to males (Fig. 2c-e), suggesting a role of $(2 \mathrm{~S}, 6 \mathrm{~S} ; 2 \mathrm{R}, 6 \mathrm{R})$ - 
HNK in the antidepressant effects of ketamine. To directly determine if metabolism of ketamine to $(2 \mathrm{~S}, 6 \mathrm{~S} ; 2 \mathrm{R}, 6 \mathrm{R})$-HNK is required for its antidepressant actions, we deuterated ketamine at the $\mathrm{C} 6$ position (6,6-dideuteroketamine; Extended Data Fig. 2f). This alteration would not change the pharmacological properties of un-metabolised ketamine, but may change the relative rate of metabolism ${ }^{23}$. Indeed, 6,6-dideuteroketamine did not change NMDAR binding affinity (Extended Data Fig. 2g), or NMDAR-mediated hyperlocomotion (Extended Data Fig. 4c,d), but robustly hindered its metabolism to (2S,6S;2R,6R)-HNK, without changes to the levels of ketamine in the brain (Fig. 2f-h). Unlike ketamine, administration of 6,6-dideuteroketamine did not induce antidepressant actions in the FST (Fig. 2i) or LH (Fig. 2j) 24 hours after administration, indicating a critical role of (2S,6S;2R, 6R)-HNK in ketamine's sustained antidepressant effects. Notably, published human data reveal a positive correlation between the antidepressant responses of ketamine and plasma (2S,6S;2R,6R)-HNK metabolite levels ${ }^{19}$.

To determine whether (2S,6S)- or (2R,6R)-HNK exert antidepressant effects independent of ketamine administration, we compared their behavioural effects in the 24-hour (sustained) FST and LH paradigms. We observed more potent antidepressant effects following administration of the (2R,6R)-HNK metabolite (Fig. 2k,l), which is exclusively derived from (R)-ketamine, and thus consistent with the greater antidepressant actions of (R)-ketamine relative to (S)-ketamine (Fig. 1b-d). Moreover, (2R,6R)-HNK resulted in a dose-dependent antidepressant action in the LH, FST and NSF tests (Extended Data Fig. 5a,c,f). We note that $(2 \mathrm{~S}, 6 \mathrm{~S})-\mathrm{HNK}$ does also exert antidepressant actions at higher doses (Extended Data Fig. $5 b, d)$. The greater antidepressant effects of (2R,6R)-HNK do not result from higher brain levels of the drug compared to (2S,6S)-HNK (Extended Data Fig. 5e). Similar to ketamine, a single (2R,6R)-HNK administration induced persistent antidepressant effects in the FST, lasting for at least three days (Extended Data Fig. 5g). A single administration of (2R,6R)HNK also reversed chronic corticosterone-induced anhedonia as assessed in the sucrose preference and female urine sniffing behavioural tasks (Extended Data Fig. 5h,i), as well as social avoidance induced by chronic social defeat stress (Fig. 2m; Extended Data Fig. 5j,k).

\section{(2R,6R)-HNK effects on glutamate receptors}

A prominent hypothesis for ketamine's mechanism of action is that it acts via direct inhibition of NMDARs localized to interneurons. This is hypothesized to lead to disinhibition of glutamatergic neurons, which receive input from interneurons, and a resultant rapid increase in glutamate synaptic transmission in mood-relevant brain regions ${ }^{24}$. However, in contrast to ketamine, $(2 \mathrm{R}, 6 \mathrm{R})-\mathrm{HNK}$ does not displace $\left[{ }^{3} \mathrm{H}\right]-\mathrm{MK}-801$ binding to the NMDAR in vitro (Fig. 3a; also see ${ }^{12}$ ) and does not functionally inhibit NMDARs localized to stratum radiatum interneurons in hippocampal slices (Fig. 2b,c). Instead, (2R,6R)-HNK induced a robust increase in AMPA receptor-mediated excitatory post-synaptic potentials (EPSPs) recorded from the CA1 region of hippocampal slices following stimulation of Schaffer collateral axons, which was sustained following washout of the drug (Fig. 3d-f). (2R,6R)-HNK also increased the frequency and amplitude of AMPA receptor-mediated excitatory post-synaptic currents (EPSCs) recorded from CA1 stratum radiatum interneurons (Extended Data Fig. 6a-j), which receive glutamatergic inputs from the Schaffer collaterals. To test the extent to which the antidepressant effect of (2R,6R)- 
HNK depends on AMPAR activation in vivo, mice were pre-treated with the AMPA receptor antagonist 2,3-dihydroxy-6-nitro-7-sulfamoyl-benzo[f]quinoxaline-2,3-dione (NBQX) 10 min prior to treatment with ketamine or $(2 \mathrm{R}, 6 \mathrm{R})$-HNK. One hour or 24 hours after the treatment mice were assessed in the FST. Similar NBQX treatment has previously been shown to prevent the antidepressant actions of ketamine, without effects on other behaviours in rodents ${ }^{15,25-27}$. Treatment with NBQX, prior to (2R,6R)-HNK, prevented both the 1- and 24-hour antidepressant effects of (2R,6R)-HNK (Fig. 3g,h), indicating that its antidepressant actions require the acute activation of AMPA receptors.

A non-invasive method used to assess ketamine-activated circuitry in both humans and rodents is the quantitative electroencephalography (qEEG) measurement of gamma-band power, which is dependent upon activation of fast ionotropic excitatory receptors, including AMPA receptors ${ }^{28-30}$. We show that, similar to ketamine, (2R,6R)-HNK administration acutely increases gamma power measured via surface electrodes in vivo (Fig. 3i,j), independent of locomotor activity changes, and without altering alpha, beta, delta or theta oscillations (Extended Data Fig. 7a-e). Importantly, pre-treatment with NBQX prevented (2R,6R)-HNK-induced increases in gamma power, thus further implicating AMPA receptors in (2R,6R)-HNK mechanism of action (Extended Data Fig. 7f-k), and validating a potential human translational biomarker of the CNS response to (2R,6R)-HNK.

Evidence indicates that mTOR signaling ${ }^{25}$, protein synthesis through eEF2 dephosphorylation ${ }^{16}$, as well as BDNF increases ${ }^{16,31}$, underlie ketamine's antidepressant responses. We examined whether administration of (2R,6R)-HNK affects phosphorylation of mTOR (Ser 2448) and eEF2 (Thr 56), or BDNF levels in synaptoneurosome fractions of the hippocampus and prefrontal cortex. No differences were observed in mTOR phosphorylation following administration of ketamine or (2R,6R)-HNK in the hippocampus or the prefrontal cortex of mice (Extended Data Fig. 8a,b;c,d). However, ketamine induced a decrease in eEF2 phosphorylation in the hippocampus (but not the prefrontal cortex) 1 and 24 hours post-injection, and increased hippocampal BDNF at 24 hours (Fig. 4a-d). These effects were recapitulated by (2R,6R)-HNK administration (Fig. 4a-d; Extended Data Fig. 8e-h), and may be partially responsible for its sustained antidepressant actions.

It is noteworthy that (2R,6R)-HNK resulted in antidepressant actions (Fig. 2k-m; Extended Data Fig. 5) at time points (e.g. 24 hours) past when its brain levels are below detectable limits (e.g. 2 hours; Extended Data Fig. 5e). Synaptic plasticity changes involving AMPA receptors are thought to underlie such long-term antidepressant actions of ketamine ${ }^{24,27}$. Here we show that while neither ketamine nor $(2 \mathrm{R}, 6 \mathrm{R})$-HNK administration altered the levels of GluA1 and GluA2 in hippocampal synaptoneurosomes 1 hour post-treatment (Fig. 4e), they both increased GluA1 and GluA2 levels 24 hours post-treatment in mouse hippocampal (Fig. 4f), but not prefrontal cortex synaptoneurosomes (Extended Data Fig. $8 \mathrm{i}, \mathrm{j})$. Consistent with an increase in synaptic AMPA receptors being involved in the sustained, 24-hour, antidepressant actions, administration of NBQX thirty minutes prior to the 24-hour FST (23.5 hours after antidepressant treatment; see timeline Fig. 4g) prevented the antidepressant actions of both ketamine and (2R,6R)-HNK (Fig. 4h). These findings implicate an AMPA receptor-mediated maintenance of synaptic potentiation to underlie the sustained antidepressant effects of (2R,6R)-HNK. 


\section{(2R,6R)-HNK lacks ketamine's side effects}

Ketamine has abuse potential, as well as sensory-dissociation properties and other sideeffects, which limit its potential widespread use for the treatment of depression ${ }^{9}$. While administration of ketamine (Extended Data Fig. 4a-d) and (2S,6S)-HNK (Fig. 5a) were associated with increased locomotor activity and motor incoordination (Fig. 5c,d), (2R,6R)HNK did not induce any significant change in locomotion, and did not affect coordination as measured with the accelerating rotarod test (Fig. 5b,d). We show that unlike ketamine, (2R, 6R)-HNK administration, even at high doses ( $375 \mathrm{mg} / \mathrm{kg})$, did not affect sensory gating as assessed with pre-pulse inhibition (Fig. 5e) or startle amplitude (Extended Data Fig. 9a). Non-competitive NMDAR antagonists, including ketamine and phencyclidine, produce discriminative stimulus effects in drug discrimination protocols and manifest cross-drug substitution profiles at an antidepressant-relevant dose range ${ }^{32}$. In ketamine-trained mice, (2R,6R)-HNK administration did not produce ketamine-related discrimination responses, whereas phencyclidine (PCP) did (Fig. 5f,g) without either of these drugs changing overall lever pressing response rates (Extended Data Fig. 9b,c), further supporting a non-NMDAR mechanism for $(2 \mathrm{R}, 6 \mathrm{R})$-HNK action including interoceptive effects, unlike the abused drugs ketamine and PCP. Since drug discrimination does not independently predict abuse potential per se, we further assessed the effects of ketamine and $(2 \mathrm{R}, 6 \mathrm{R})-\mathrm{HNK}$ in an intravenous drug self-administration paradigm, classically used for the evaluation of abuse/addiction liability. Intravenous ketamine was readily self-administered and resulted in a significant increase in drug intake (Fig. 5h; Extended Data Fig. 9d). In contrast, mice did not self-administer pharmacologically-relevant doses of (2R,6R)-HNK under the same conditions (Fig. 5h; Extended Data Fig. 9d). Overall, (2R,6R)-HNK administration revealed an innocuous sideeffect profile compared to ketamine.

\section{Discussion}

Our data provide new evidence explaining the unique antidepressant effects of ketamine and implicate an NMDAR inhibition-independent mechanism. These findings reveal that production of a distinct metabolite of ketamine is necessary and sufficient to produces ketamine's antidepressant actions. Overall, our data suggest that administration of $(2 R, 6 R)-$ HNK induces an acute increase in glutamatergic signaling (as supported by our EPSP, EPSC, and qEEG measurements), followed by a long-term adaptation involving the upregulation of synaptic AMPA receptors, as evidenced by an increase in GluA1 and GluA2 in hippocampal synapses. This is supported by the finding that NBQX reverses both the acute (delivered prior to (2R,6R)-HNK) (Fig. 3g,h) and sustained (delivered after (2R,6R)-HNK; Fig 4g,h) antidepressant actions of (2R,6R)-HNK. Considering the lack of side effects, and the favourable physiochemical properties of $\mathrm{HNKs}^{33}$, these findings have relevance for the development of next generation, rapid-acting antidepressants.

\section{Methods}

Animals

Mice and rats were acclimated to University of Maryland, Baltimore vivarium for at least seven days prior to experiments. Food and water were available ad libitum. CD-1 mice 
(males unless otherwise noted; 8-10 weeks old; Charles River Laboratories, MA, USA) were housed in groups of four-five per cage with a constant 12-hour light/dark cycle (lights on/off at 07:00/19:00). For the social defeat experiments, 8-9 week old male C57BL/6J mice (University of Maryland, Baltimore veterinary resources breeding colony) and retired male CD-1 breeders (Charles River Laboratories, MA, USA) were used. For the whole-cell and field potential electrophysiological recordings, male Sprague-Dawley rats (postnatal day 24-35; Charles River, Wilmington, MA) were used. All experimental procedures were approved by the University of Maryland, Baltimore Animal Care and Use Committee and were conducted in full accordance with the National Institutes of Health Guide for the Care and Use of Laboratory Animals.

\section{Drugs}

(R,S)-ketamine, (S)-ketamine, desipramine, MK-801, phencyclidine (PCP) (Sigma-Aldrich, St. Louis, MO, USA), (R)-ketamine (Cayman Chemicals, Ann Arbor, MI, USA) and NBQX (National Institute of Mental Health Chemical Synthesis and Drug Supply Program) were dissolved in $0.9 \%$ saline. (2S,6S)-HNK, $(2 \mathrm{R}, 6 \mathrm{R})-\mathrm{HNK}$, and 6,6-dideuteroketamine hydrochloride were synthesised and characterised both internally at the National Center for Advancing Translational Sciences and at SRI International (Menlo Park, CA, USA) as described in Supplementary Information. Absolute and relative stereochemistry for (2S,6S)HNK and (2R,6R)-HNK were confirmed by small molecule $\mathrm{x}$-ray crystallography, as described in the Supplementary Information.

All drugs were dissolved in $0.9 \%$ saline, and administered intraperitoneally (i.p.) in a volume of $7.5 \mathrm{ml} / \mathrm{kg}$ of body mass by a male experimenter for the behavioural studies. Corticosterone (4-pregnen-11 $\beta$, 21-diol-3, 20-dione 21-hemisuccinate; Steraloids, Newport, RI, USA) was dissolved in tap water. For the electrophysiology recordings, test drugs were diluted in artificial cerebrospinal fluid (ACSF).

\section{Forced-swim test (FST)}

Mice were tested in the FST 1 hour and/or 24 hours post-injection. During the FST, mice were subjected to a 6-min swim session in clear Plexiglass cylinders $(30 \mathrm{~cm}$ height $\times 20 \mathrm{~cm}$ diameter) filled with $15 \mathrm{~cm}$ of water $\left(23 \pm 1^{\circ} \mathrm{C}\right)$. The FST was performed in normal light conditions (800 Lux). Sessions were recorded using a digital video-camera. Immobility time, defined as passive floating with no additional activity other than that necessary to keep the animal's head above the water, was scored for the last 4 min of the 6-min test by a trained observer.

We conducted three different FST experiments where we utilized the AMPA receptor antagonist NBQX. In the first two experiments, we administered NBQX 10 minutes prior to ketamine, (2R,6R)-hydroxynorketamine, or vehicle and then tested the mice 1 hour and 24 hours later to assessed whether AMPA receptor activity is necessary for the acute actions of these drugs, leading to both acute and sustained antidepressant effects. In the third experiment we first administered ketamine, (2R,6R)-HNK, or vehicle and then, 23.5 hours later mice received either NBQX or vehicle. Thirty minutes later we tested these mice in the FST, to assess effects of AMPA receptor activity on sustained antidepressant actions. 


\section{Open-field test}

Mice were placed into individual open-field arenas $(50 \mathrm{~cm}$ length $\times 50 \mathrm{~cm}$ width $\times 38 \mathrm{~cm}$ height; San Diego Instruments, San Diego, CA, USA) for a 60-min habituation period. Mice then received an injection of the respective drug and assessed for locomotor activity for another $60 \mathrm{~min}$. Distance travelled was analysed using TopScan v2.0 (CleverSys, Inc, Reston, VA, USA).

\section{Novelty-suppressed feeding (NSF)}

Mice were singly housed and food-deprived for twenty-four hours in freshly-made homecages. Two normal chow diet pellets were placed on an inverted weighing-boat platform (10 $\times 10 \times 1.5 \mathrm{~cm})$ in the center of an open-field arena $(40 \times 40 \mathrm{~cm})$. Thirty or sixty min (see Figure captions) after drug administration, mice were introduced into a corner of the arena. The time needed for the mice to take a bite of food was recorded over a 10-min period by a trained observer. After the test, the mice were returned to their home cage containing preweighed food pellets, and latency to start biting the pellet, as well as consumption was recorded for a period of $10 \mathrm{~min}$. There was no significant change in home cage latency or consumption in any of our experiments (data not shown).

\section{Learned helplessness (LH)}

The LH paradigm consisted of three different phases: inescapable shock training, LH screening, and the LH test. For the inescapable shock portion of the test (Day 1), the animals were placed in one side of two-chambered shuttle boxes $(34 \mathrm{~cm}$ height $\times 37 \mathrm{~cm}$ width $\times 18$ $\mathrm{cm}$ depth; Coulbourn Instruments, PA, USA), with the door between the chambers closed. Following a five-min adaptation period, 120 inescapable foot-shocks $(0.45 \mathrm{~mA}, 15 \mathrm{sec}$ duration, randomised average inter-shock interval of $45 \mathrm{sec}$ ) were delivered through the floor. During the screening session (Day 2), the mice were placed in one of the two chambers of the apparatus for $5 \mathrm{~min}$. A shock $(0.45 \mathrm{~mA})$ was then delivered, and the door between the two chambers was raised simultaneously. Crossing over into the second chamber terminated the shock. If the animal did not cross over, the shock terminated after 3 sec. A total of 30 screening trials of escapable shocks were presented to each mouse with an average of 30-sec delay between each trial. Mice that developed helplessness behaviour ( $>5$ escape failures during the last 10 screening shocks) received the assigned drug 24 hours following screening (Day 3). During the LH test phase (Day 4), the animals were placed in the shuttle boxes and, after a 5-min adaptation period, a $0.45 \mathrm{~mA}$ shock was delivered concomitantly with door opening for the first five trials, followed by a 2-sec delay for the next 40 trials. Crossing over to the second chamber terminated the shock. If the animal did not cross over to the other chamber, the shock was terminated after $24 \mathrm{sec}$. A total of 45 trials of escapable shocks were presented to each mouse with 30-sec inter-trial intervals. The number of escape failures was recorded for each mouse by an automated computer software (Graphic State v3.1; Coulbourn Instruments, Whitehall, PA, USA).

\section{Chronic social defeat stress and social interaction}

The timeline for the social defeat experiments is presented in Extended Data Fig. 3a. Male C57BL/6J mice underwent a 10-day chronic social defeat stress paradigm, as described 
elsewhere ${ }^{34}$, with some modifications. Briefly, experimental mice were introduced to the home cage ( $43 \mathrm{~cm}$ length $\times 11 \mathrm{~cm}$ width $\times 20 \mathrm{~cm}$ height) of a resident aggressive retired CD-1 breeder, prescreened for aggressive behaviours, for $10 \mathrm{~min}$. Following this physical attack phase, mice were transferred and housed in the opposite side of the resident's cage divided by a Plexiglas perforated divider, in order to maintain continuous sensory contact. This process was repeated for 10 days. Experimental mice were introduced to a novel aggressive CD-1 mouse each day. On day 11 , test mice were screened for susceptibility in a social interaction/avoidance choice test. The social interaction apparatus consisted of a rectangular three-chambered box (mouse conditioned-place preference chamber; Stoelting Co., Wood Dale, IL, USA), see Extended Data Fig.3b) comprised of two equal sized endchambers and a smaller central chamber. The social interaction/avoidance choice test consisted of two 5-min phases. During the habituation phase, mice explored the empty apparatus. During the test phase, two small wire cages (Galaxy Cup, Spectrum Diversified Designs, Inc., Streetsboro, OH, USA), one containing a "stranger" CD-1 mouse and the other one empty, were placed in the far corners of each chamber. The time spent interacting (nose within close proximity of the cage) with the "stranger" mouse versus the empty cage was analysed using TopScan video tracking software (CleverSys, Reston, Virginia).

Locomotor activity (total distance moved over $5 \mathrm{~min}$ ) and number of total crosses into and out of the central chamber were also measured. The social interaction ratio was calculated by dividing the time spent interacting with the "stranger" by the time spent with the empty cage. Mice having a social interaction ratio higher than 1.0 were considered resilient and mice with a social interaction ratio lower than 1.0 were considered susceptible. On day 13 resilient and susceptible mice received an i.p. injection of either saline, (R,S)-KET (20 $\mathrm{mg} / \mathrm{kg}$; chosen based upon dose previously effective in C57BL/6J mice $\left.{ }^{34}\right)$, MK-801 (0.1 $\mathrm{mg} / \mathrm{kg}$ ) or (2R,6R)-HNK (20 mg/kg). Mice were re-tested for social interaction/avoidance on day 15 (24 hours following treatment).

\section{Chronic corticosterone-induced anhedonia}

Sucrose preference test-For assessing the baseline sucrose preference, mice were singly housed for 24 hours and presented with two identical bottles containing either tap water or $1 \%$ sucrose solution. Following baseline sucrose measurement, mice were re-group housed ( 5 mice per cage) and treated for 4 weeks with corticosterone $(25 \mu \mathrm{g} / \mathrm{ml}$ equivalent) given in water bottles. Prior to initiation of any behavioural measurements, animals were weaned off corticosterone treatment; 3 days corticosterone $12.5 \mu \mathrm{g} / \mathrm{ml}$ and 3 days corticosterone $6.25 \mu \mathrm{g} / \mathrm{ml}$, followed by 1 week of complete withdrawal. Mice were subsequently singly-housed in freshly-made home cages and provided with two bottles containing either tap water or $1 \%$ sucrose solution. Twenty-four hours later, mice that developed the anhedonia phenotype ( $<70 \%$ sucrose preference) were treated with saline or $(2 \mathrm{R}, 6 \mathrm{R})-\mathrm{HNK}(10 \mathrm{mg} / \mathrm{kg})$ and sucrose preference was measured after an additional 24 hours.

Female urine sniffing test-A separate cohort of mice was treated with the same chronic corticosterone administration paradigm as described above but assessed for female urine sniffing preference as a measure of hedonic behaviour ${ }^{35}$. Mice were singly-housed in freshly-made home cages for a habituation period of $10 \mathrm{~min}$. Subsequently, one plain cotton 
tip was secured on the center of the cage wall and mice were allowed to sniff and habituate to the tip for a period of $30 \mathrm{~min}$. Then, the plain cotton tip was removed and replaced by two cotton tip applicators, one infused with fresh female mouse estrus urine and the other with fresh male mouse urine. These applicators were presented and secured at the two corners of the cage wall simultaneously. Sniffing time for both female and male urine was scored by a trained observer for a period of three min. Twenty-four hours later, mice that developed anhedonia phenotype ( $<65 \%$ female urine preference; susceptible phenotype), as well as mice that did not develop the anhedonia phenotype (>75\% female urine preference; resilient phenotype) were treated with either saline or (2R,6R)-HNK (10 mg/kg). Mice were re-tested for female urine preference 24 hours later.

\section{Pre-pulse inhibition (PPI)}

Mice were individually tested in acoustic startle boxes (SR-LAB, San Diego Instruments). Following drug administration, mice were placed in the startle chamber for a 30-min habituation period. The experiment started with a further 5-min adaptation period during which the mice were exposed to a constant background noise $(67 \mathrm{~dB})$, followed by five initial startle stimuli ( $120 \mathrm{~dB}, 40 \mathrm{msec}$ duration each). Subsequently, animals were exposed to four different trial types: pulse alone trials ( $120 \mathrm{~dB}, 40 \mathrm{msec}$ duration), three pre-pulse trials of 76 and 81 of white noise bursts ( $20 \mathrm{msec}$ duration) preceding a $120 \mathrm{~dB}$ pulse by 100 msec, and background $(67 \mathrm{~dB})$ no-stimuli trials. Each of these trials was randomly presented five times. The dose of ketamine $(30 \mathrm{mg} / \mathrm{kg}$ ) was selected based on a dose-response experiment we performed in a previous study ${ }^{36}$. The $\%$ pre-pulse inhibition (PPI) was calculated using the following formula: [(magnitude on pulse alone trial - magnitude on prepulse + pulse trial) $/$ magnitude on pulse alone trial $] \times 100$.

\section{Drug discrimination}

Mice were food-restricted until they reached $85 \%$ of their initial body weight and were maintained at $85 \%$ throughout the duration of the experiment. Animals were trained to lever press for food (20 mg sucrose pellets; TestDiet, St. Luis, MO, USA) in standard two-lever operant conditioning chambers (Coulbourn Instruments, Whitehall, PA, USA), under a fixed-ratio 5 schedule of reinforcement (FR5) in daily 30-min sessions. After stable responding was maintained over 3 consecutive sessions, mice were trained to discriminate ketamine $(10 \mathrm{mg} / \mathrm{kg})$ from saline under a double alternation schedule (i.e., ketamine, ketamine, saline, saline), which required on average 40 training sessions. Mice received either ketamine $(10 \mathrm{mg} / \mathrm{kg}$; i.p.) or saline $15 \mathrm{~min}$ prior to the start of the 30-min session. Responding to the correct lever resulted in the delivery of a reward, while incorrect responding reset the FR for correct lever-responding. Drug discrimination test sessions were conducted when mice reached the following criteria: (1) first FR5 completed on the correct lever, and (2) $285 \%$ correct lever responding over the entire session. During the 30 -min test sessions mice received either saline, ketamine $(10 \mathrm{mg} / \mathrm{kg})$, phencyclidine (PCP; $3 \mathrm{mg} / \mathrm{kg}$ ) or (2R,6R)-HNK (10 and $50 \mathrm{mg} / \mathrm{kg}$ ). At this stage, completion of the FR5 schedule on either lever resulted in the delivery of food reward. Lever response and pellet delivery were monitored and controlled by an automated computer system (Graphic State v3.1; Coulbourn Instruments, Whitehall, PA, USA). 


\section{Intravenous drug self-administration}

Apparatus-Each operant chamber was equipped with one lever, a dipper liquid delivery system, a 22ga. liquid swivel and a syringe pump (located outside the chamber). The lever was a balanced rocker arm that broke an infrared photo beam when $0.5 \mathrm{~g}$ of force was applied. Two stimulus lights were used; one was positioned to illuminate the translucent lever and the other was positioned above the liquid delivery recess. The lever light was illuminated during periods of water or drug availability; the second light was illuminated during water or drug delivery. The system was controlled by an integrated Coulbourn (Whitehall, PA) environmental control system and Med Associates interface (St. Albans, VT).

Water Training-Mice were first trained to complete an operant response for water reinforcement. Completion of the response requirements on the lever illuminated stimulus lights above a spout and delivered a small amount of water. Initially, the response requirement was one lever press (FR1); after completion of each 50 reinforcements the fixed ratio requirement was increased by one $(F R X+1)$ to a maximum of FR4. Mice were trained 24 hours/day for four days with free access to food.

Surgery-Following completion of the water training, mice were surgically prepared with a catheter implanted in the jugular vein. Surgical procedures were performed under ketamine- (90 mg/kg, i.p.) and xylazine- (16 mg/kg, i.p.) induced anaesthesia. Silastic tubing (0.012" i.d.) was implanted in the right jugular vein to the level of the atrium, passed subcutaneously and exited in the midscapular region. The catheter was connected to a tether/ swivel system that was mounted to the skull of the mouse with dental cement.

IV Drug Self-administration-Seven days post-surgery, mice were placed in the operant chamber and given access (FR4) to $0.32,1.0,3.2$, or 0 (saline) $\mathrm{mg} / \mathrm{kg}$ drug per infusion for 5 days at each dose. Completion of each FR resulted in the illumination of the

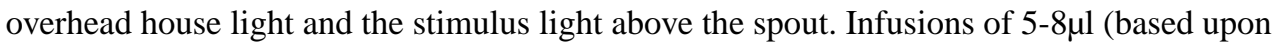
body weight) were given over a period of $15 \mathrm{sec}$. A $30 \mathrm{sec}$ time-out period, during which house and stimulus lights were out, followed the completion of each infusion. Each mouse had access to drug for 6 hours/day and free access to food and water 24 hours/day. A 12hour light/dark cycle was maintained (lights on/off at 07:00/19:00). Each animal remained in its operant chamber for the duration of the experiment. A stimulus light illuminating the lever signaled drug availability. Only those animals with patent catheters at the end of the experiment were included in the analysis. The average number of reinforcements and drug intake during the last three days at each dose were used as dependent measures.

\section{Rotarod}

The rotarod test was conducted to compare the effects of ketamine, $(2 \mathrm{~S}, 6 \mathrm{~S})-\mathrm{HNK}$ and $(2 \mathrm{R}$, 6R)-HNK on motor coordination. The experiment consisted of two phases: training phase (4 days) and a test phase (1 day). On each of the training days five trials (trial time: $3 \mathrm{~min}$ ) were conducted with an inter-trial interval of two min Mice were individually placed on the rotarod apparatus (IITC Life Science; Woodland Hills, CA, USA) and the rotor (3.75 inch diameter) accelerated from 5-20 RPM over a period of three minutes. Latency to fall was 
recorded for each trial. Animals with an average of $<100 \mathrm{sec}$ of latency to fall during the last training day were excluded from the experiment. On the test day (day 5), mice received (i.p.) injections of saline, (R,S)-KET (10 mg/kg), (2S,6S)-HNK (25 or $125 \mathrm{mg} / \mathrm{kg})$ or $(2 \mathrm{R}, 6 \mathrm{R})$ HNK ( 25 or $125 \mathrm{mg} / \mathrm{kg}$ ) and were tested in the rotating rod 5-, 10-, 15-, 20-, 30- and 60-min post-injection using the same procedure described for the training days.

\section{Tissue distribution and clearance measurements of ketamine and metabolites}

At 10, 30, 60, or 240 minutes following drug administration mice were exposed to 3\% isoflurane and subsequently decapitated. Trunk blood was collected in EDTA-containing tubes and centrifuged at $5938 \times \mathrm{g}$ for $6 \min \left(4^{\circ} \mathrm{C}\right)$. Plasma was collected and stored at $-80^{\circ} \mathrm{C}$ until analysis. Whole brains were simultaneously collected, rinsed with phosphate-buffered saline, immediately frozen in dry ice and stored at $-80^{\circ} \mathrm{C}$ until analysis.

The concentrations of $(\mathrm{R}, \mathrm{S})$-ketamine and 6,6-dideuteroketamine ((R,S)- $\mathrm{d}_{2}$-ketamine) and their respective metabolites in plasma and brain tissue were determined by achiral liquid chromatography-tandem mass spectrometry following a previously described method ${ }^{33}$, with slight modifications. The analysis was accomplished using an Eclipse XDB-C18 guard column $(4.6 \mathrm{~mm} \times 12.5 \mathrm{~mm})$ and a Varian Pursuit XRs 5 C18 analytical column $(250 \mathrm{~mm} \times$ $4.0 \mathrm{~mm}$ ID, $5 \mu \mathrm{m}$; Varian, Palo Alto, CA, USA). The mobile phase consisted of ammonium acetate [5 mM, pH 7.6] as component $\mathrm{A}$ and acetonitrile as component $\mathrm{B}$. A linear gradient was run as follows: $0 \min 20 \% \mathrm{~B} ; 5 \min 20 \% \mathrm{~B} ; 15 \min 80 \% \mathrm{~B} ; 20 \mathrm{~min} 20 \% \mathrm{~B}$ at a flow rate of $0.4 \mathrm{ml} / \mathrm{min}$. The total run time was $30 \mathrm{~min}$ per sample. For plasma and brain samples, the calibration standards ranged from $10,000 \mathrm{ng} / \mathrm{ml}$ to $19.5 \mathrm{ng} / \mathrm{ml}$ for (R,S)-ketamine, (R,S)norketamine, (2R,6R;2S,6S)-HNK, (R,S)-dehydronorketamine, (R,S)- $\mathrm{d}_{2}$-ketamine, (R,S)-- $\mathrm{d}_{2}$ norketamine and d-(2S,6S;2R,6R)-HNK. The quantitation of (R,S)-ketamine, $(\mathrm{R}, \mathrm{S})-\mathrm{d}_{2^{-}}$ ketamine and their respective metabolites was accomplished by calculating area ratios using $\mathrm{d}_{4}$-ketamine (10 $\mu \mathrm{l} \mathrm{of} 10 \mu \mathrm{g} / \mathrm{ml}$ solution) as the internal standard. The MS/MS analysis was performed using a triple quadrupole mass spectrometer model API 4000 system from Applied Biosystems/MDS Sciex equipped with Turbo Ion Spray® (TIS) (Applied Biosystems, Foster City, CA, USA). The data was acquired and analysed using Analyst version 1.4.2 (Applied Biosystems). Positive electrospray ionization data were acquired using multiple reaction monitoring (MRM) using the following transitions for (R,S)ketamine studies: $238 \rightarrow 125$ (ketamine); $224 \rightarrow 125$ (norketamine); $222 \rightarrow 177$ (dehydronorketamine); $240 \rightarrow 125$ (HNK); $254 \rightarrow 151(\mathrm{HK})$ and (R,S)- $\mathrm{d}_{2}$-ketamine studies: $240 \rightarrow 125\left(\mathrm{~d}_{2}\right.$-ketamine); $226 \rightarrow 125\left(\mathrm{~d}_{2}\right.$-norketamine); $223 \rightarrow 178(\mathrm{~d}$ dehydronorketamine); $241 \rightarrow 125$ (d-(2,6)-HNK); 242-125 (d $\left.\mathrm{d}_{2}-(2,5)-\mathrm{HNK}\right) ;\left(\mathrm{d}_{2}-(2,4)-\mathrm{HNK}\right)$ and $255 \rightarrow 151$ (d-(2,6)-HK).

\section{MK-801 displacement binding}

NMDAR binding assays were performed according to Chiu et al., $1999^{37}$, with minor modifications. Rat brains were homogenised and membrane fractions were collected. Aliquoted membranes were stored at $-80^{\circ} \mathrm{C}$ until use. Membrane pellets were washed 5 times with an ice-cold buffer (20 mM HEPES, $1 \mathrm{mM}$ EDTA, pH 7.0) before use. The binding assays were set up in 96-well plates using $5 \mathrm{nM}\left[{ }^{3} \mathrm{H}\right]-\mathrm{MK}-801$ and rat brain membranes (100 $\mu \mathrm{g} / \mathrm{well})$ in a final volume of $125 \mu \mathrm{l} /$ well in the NMDAR binding buffer (20 
mM HEPES, 1 mM EDTA, $100 \mu$ M Glutamate, $100 \mu \mathrm{M}$ Glycine, $\mathrm{pH}$ 7.0). Test compounds were first distributed in 96 -well plates $(25 \mu \mathrm{l} /$ well at $5 \times$ of final concentrations ranging from $0.1 \mathrm{nM}$ to $10 \mu \mathrm{M}, 11$ points) in triplicate. The radioligand, $\left[{ }^{3} \mathrm{H}\right]-\mathrm{MK}-801$ was added $(50 \mu \mathrm{l} /$ well at $2.5 \times$ of final $5 \mathrm{nM}$ ) to all wells. Reactions started with the addition of $50 \mu \mathrm{l}$ rat brain membrane and were incubated for 1 hour in the dark at room temperature. The reactions were harvested via rapid filtration onto Whatman GF/B glass fiber filters pre-soaked with $0.3 \%$ polyethyleneimine using a 96-well Brandel harvester, followed by three quick washes each with $500 \mu \mathrm{l}$ chilled wash buffer ( $50 \mathrm{mM}$ Tris $\mathrm{HCl}, \mathrm{pH}$ 7.4). Filters were microwavedried and scintillation cocktail was then melted onto the filter mates on a hot plate. The radioactivity retained on the filters was counted in a MicroBeta scintillation counter. All assays were performed in triplicates.

\section{Western blots}

To purify synaptoneurosomes, mouse prefrontal cortex and hippocampi were dissected and homogenised in Syn-PER Reagent (ThermoFisher Scientific, Waltham, MA, USA; Cat \# 87793) with $1 X$ protease and phosphatase inhibitor cocktail (ThermoFisher Scientific, Waltham, MA, USA; Cat \# 78440). The homogenate was centrifuged for $10 \mathrm{~min}$ at 1,200 $\times$ g at $4{ }^{\circ} \mathrm{C}$. The supernatant was centrifuged at $15,000 \times \mathrm{g}$ for 20 min at $4{ }^{\circ} \mathrm{C}$. After centrifugation, the pellet (synaptosomal fraction) was re-suspended and sonicated in N-PER Neuronal Protein Extraction Reagent (ThermoFisher Scientific, Waltham, MA, USA; Cat \# 87792). Protein concentration was determined via the BCA protein assay kit (ThermoFisher Scientific, Waltham, MA, USA; Cat \# 23227). Equal amount of proteins (10-40 $\mu \mathrm{g}$ as optimal for each antibody) for each sample was loaded into NuPage 4-12\% Bis-Tris gel for electrophoresis. Gel transfer was performed with the TransBlot Turbo Transfer System (BioRad, Hercules, CA, USA) Nitrocellulose membranes with transferred proteins were blocked with 5\% milk in TBST (TBS $+0.1 \%$ Tween-20) for 1 hour and kept with primary antibodies overnight at $4{ }^{\circ} \mathrm{C}$. The following primary antibodies were used: phospho-eEF2 (at Thr56; Cell Signaling Technology, Danvers, MA, USA; Cat \# 2331), total eEF2 (Cell Signaling Technology, Danvers, MA, USA; Cat \# 2332), phospho-mTOR (at Ser2448; Cell Signaling Technology, Danvers, MA, USA; Cat \# 2971), total mTOR (Cell Signaling Technology, Danvers, MA, USA; Cat \# 2983), GluA1 (Cell Signaling Technology, Danvers, MA, USA; Cat \# 2983), GluA2 (Cell Signaling Technology, Danvers, MA, USA; Cat \# 13607), BDNF (Santa Cruz Biotechnology, Dallas, Texas, USA; Cat \# sc-546), and GAPDH (Abcam, Cambridge, MA, USA; Cat \# ab8245). The next day, blots were washed three times in TBST and incubated with horseradish peroxidase conjugated anti-mouse or anti-rabbit secondary antibody (1:5000 to 1:10000) for 1 hour. After final three washes with TBST, bands were detected using enhanced chemiluminescence (ECL) with the Syngene Imaging System (G:Box ChemiXX9). After imaging, the blots were incubated in the stripping buffer (ThermoFisher Scientific, Waltham, MA, USA; Cat \# 46430) for 10-15 min at room temperature followed by three time washes with TBST. The stripped blots were incubated in blocking solution for 1 hour and incubated with the primary antibody directed against total levels of the respective protein or GAPDH for loading control. Densitometric analysis of phospho- and total immunoreactive bands for each protein was conducted using Syngene's GenTools software. The values for the phosphorylated forms of proteins were normalised to phosphorylation-independent levels of the same protein. Phosphorylation-independent levels 
of proteins were normalised to GAPDH. Fold change was calculated by normalisation to saline-treated control group for each protein or phosphoprotein.

\section{Electroencephalogram (EEG)}

Surgery-EEG experiments were performed according to Raver et al. ${ }^{38}$, with minor modifications. Mice were anaesthetised with isoflurane (3.5\%) and maintained under anaesthesia (2-2.5\%) throughout the surgery. Mice received analgesia (carprofen, $5 \mathrm{mg} / \mathrm{kg}$, i.p.) prior to the start of surgery. An F20-EET radio-telemetric transmitter (Data Sciences International, Minneapolis, MN, USA) was placed subcutaneously and its leads implanted over the dura above the frontal cortex (1.7 mm anterior to bregma) and the cerebellum (6.4 $\mathrm{mm}$ posterior to bregma). Animals recovered from surgery for 7 days prior to recordings.

EEG recordings-For comparisons between saline, ketamine, and (2R,6R)-HNK, mice were singly housed and acclimated to the behavioural room for 24 hours prior to EEG recordings. EEGs were recorded using the Dataquest A.R.T. acquisition system (Data Sciences International, Minneapolis, MN, USA) with frontal EEG recordings referenced to the cerebellum. Baseline EEG $(30 \mathrm{~min})$ recordings were followed by an i.p. injection of saline, ketamine $(10 \mathrm{mg} / \mathrm{kg})$ or $(2 \mathrm{R}, 6 \mathrm{R})-\mathrm{HNK}(10 \mathrm{mg} / \mathrm{kg})$ and a further $60 \mathrm{~min}$ of postinjection recordings. To assess effects of NBQX on (2R,6R)-HNK-induced changes in EEG oscillations, mice were acclimated to the behavioural room 1.5-2 hours prior to recordings. Baseline (30 min) recordings were followed by an i.p. injection of either saline or NBQX $(10 \mathrm{mg} / \mathrm{kg}$ ) and $30 \mathrm{~min}$ later mice received an injection (i.p.) of (2R,6R)-HNK $(10 \mathrm{mg} / \mathrm{kg})$ and recordings continued for $60 \mathrm{~min}$ post-injection.

In Vivo Data Analysis-EEGs were analysed using custom-written MATLAB scripts (Version 2012a, Mathworks, MA) and the mtspecgramc routine in the Chronux Toolbox (http://chronux.org ${ }^{39}$ ). Oscillation power in each bandwidth (delta $=1-3 \mathrm{~Hz}$; theta=4-7 Hz; alpha $=8-12 \mathrm{~Hz}$; beta $=13-29 \mathrm{~Hz}$; gamma $=30-80 \mathrm{~Hz}$ ) was computed in 10-min bins from spectrograms for each animal.

\section{Field recordings}

Removal of the rat brains, as well as dissection of the hippocampi were performed in icecold artificial cerebrospinal fluid (ACSF) bubbled with $95 \% \mathrm{O}_{2} / 5 \% \mathrm{CO}_{2}$. The ACSF contained in mM: $124 \mathrm{NaCl}, 3 \mathrm{KCl}, 1.25 \mathrm{NaH}_{2} \mathrm{PO}_{4}, 1.5 \mathrm{MgCl}_{2}, 2.5 \mathrm{CaCl}_{2}, 26 \mathrm{NaHCO}_{3}$, and 10 glucose. Hippocampal slices were cut at $400 \mu \mathrm{m}$ using a vibratome and kept in a holding chamber at the interface of ACSF and humidified $95 \% \mathrm{O}_{2} / 5 \% \mathrm{CO}_{2}$ for at least 1 hour. For the field excitatory postsynaptic potentials (fEPSPs), slices were transferred to a submersion-type recording chamber and perfused with ACSF (1-2 $\mathrm{ml} / \mathrm{min}$; room temperature). Picrotoxin $(0.1 \mathrm{mM})$, CGP52432 $(2 \mu \mathrm{M})$ and APV $(80 \mu \mathrm{M})$ were added to block $\mathrm{GABA}_{\mathrm{A}}, \mathrm{GABA}_{\mathrm{B}}$ and NMDA receptors respectively. Concentric bipolar tungsten electrodes were placed in stratum radiatum to stimulate the Schaffer collateral (SC) afferents. Extracellular recording pipettes were filled with ACSF (3-5 M $\Omega$ ) and placed in stratum radiatum of area CA1. Field potentials were evoked by monophasic stimulation (100 $\mu$ s duration) at $0.1 \mathrm{~Hz}$. The stimulus intensity was set at $150 \%$ of threshold intensity, resulting in fEPSPs amplitude of $0.1-0.3 \mathrm{mV}$. A stable baseline was recorded for at least 10 
min. Vehicle and (2R,6R)-HNK were applied by perfusion over a period of 1 hour followed by washout using ACSF. For AMPA receptor-mediated responses, peak fEPSP amplitudes and slopes, measured over a window of 1-4 msec following the rising phase of the response, are reported as \% change from baseline. DNQX $(50 \mu \mathrm{M})$ was bath-applied to ensure AMPAmediated responses. Experiments were performed and analysed blind to treatment groups, using pCLAMP software (Molecular Devices, Sunnylvale, CA, USA).

\section{Whole-cell patch-clamp recordings}

Rats were euthanised by $\mathrm{CO}_{2}$ asphyxiation followed by decapitation. Removal of the brains, as well as dissection and slicing of the hippocampi were performed in an ice-cold solution consisting of a mixture of equal parts of regular ACSF and sucrose-containing ACSF. ACSF was composed of (in mM): $125 \mathrm{NaCl}, 26 \mathrm{NaHCO}_{3}, 2.5 \mathrm{KCl}, 1.25 \mathrm{NaH}_{2} \mathrm{PO}_{4}, 2 \mathrm{CaCl}_{2}, 1$ $\mathrm{MgCl}_{2}$, and 25 glucose. Sucrose-containing ACSF was composed of (in $\mathrm{mM}$ ): 230 sucrose, $2.5 \mathrm{KCl}, 1.25 \mathrm{NaH}_{2} \mathrm{PO}_{4}, 26 \mathrm{NaHCO}_{3}, 0.5 \mathrm{CaCl}_{2}, 10 \mathrm{MgSO}_{4}$, and 10 glucose. Hippocampal slices of 300- $\mu \mathrm{m}$ thickness were cut using a vibratome (Leica VT1000S; Leica Microsystems Inc., Bannockburn, IL) and transferred to an immersion chamber containing regular ACSF that was continuously bubbled with $95 \% \mathrm{O}_{2} / 5 \% \mathrm{CO}_{2}$ and maintained in a water bath at $30^{\circ} \mathrm{C}$.

At the time of recordings, hippocampal slices were transferred to a 1-ml recording chamber, where they were superfused at $2 \mathrm{ml} / \mathrm{min}$ with ACSF that was continuously bubbled with $95 \% \mathrm{O}_{2} / 5 \% \mathrm{CO}_{2}$. In all experiments, ACSF used to superfuse the slices contained the muscarinic antagonist atropine $(0.5 \mu \mathrm{M})$ and the $\mathrm{GABA}_{\mathrm{A}}$ receptor antagonist picrotoxin (50 $\mu \mathrm{M})$. Whole-cell patch-clamp recordings were obtained from the soma of CA1 stratum radiatum interneurons in hippocampal slices according to standard patch-clamp techniques using an EPC9 amplifier (HEKA Elektronik, Lambrecht, Germany). The signals were filtered at $3 \mathrm{kHz}$ and analysed using pCLAMP 10.3 or WinEDR v3.2.6 (University of Strathclyde, Glasgow, Scotland). The patch-clamp pipettes were pulled from a borosilicate glass capillary (1.2-mm OD) and had resistances between 3 and $5 \mathrm{M} \Omega$ when filled with internal solution. The internal pipette solution contained (in $\mathrm{mM}$ ): 10 ethylene-glycol-bis( $\beta$ amino-ethylether)- $N$ - $N$-tetraacetic acid, 10 4-(2-hydroxyethyl)-1-piperazineethanesulfonic acid, $130 \mathrm{Cs}$-methane sulfonate, $10 \mathrm{CsCl}, 2 \mathrm{MgCl}_{2}, 5$ lidocaine $\mathrm{N}$-ethyl bromide, and $0.5 \%$ biocytin ( $\mathrm{pH}$ adjusted to 7.3 with $340 \mathrm{mOsm} \mathrm{CsOH}$ ). A specially adapted U-tube developed in the Albuquerque laboratory was used to apply NMDA $(50 \mu \mathrm{M})$ to the neurons for the NMDA-evoked current experiments. NMDA-evoked currents and spontaneous AMPAmediated excitatory postsynaptic currents (sEPSCs) were recorded at $-40 \mathrm{mV}$ and $60 \mathrm{mV}$ respectively. A single neuron was studied in each slice. All experiments were carried out at room temperature $\left(20-22^{\circ} \mathrm{C}\right)$. The peak amplitude of NMDA-evoked currents was analysed using the pCLAMP v10.3 software, with baseline determined as the mean value obtained prior to drug application and the final (16 min) washout time point. Frequency and peak amplitude of AMPA sEPSCs were analysed using WinEDR v3.2.6.

\section{Statistical analyses}

Required samples sizes were estimated based upon our past experience performing similar experiments. Experimentation and analysis were performed in a manner blind to treatment 
assignments in all experiments with the exception of the whole-cell patch-clamp recordings and intravenous drug self-administration. For all blinded experiments, mice were randomly assigned to treatment groups. Statistical analyses were performed using GraphPad Prism software 16 . By pre-established criteria, values greater than $2 \pm$ S.D. from individual group means were excluded from the analyses. All statistical tests were two-tailed, and significance was assigned at $p<0.05$. Normality and equal variances between group samples were assessed using the Kolmogorov-Smirnov and Brown-Forsythe tests respectively. When normality and equal variance between sample groups was achieved, ANOVAs were followed by a Holm-Š́ídák post-hoc comparison when significance was reached, and significant results are indicated with asterisks in the Figures. As a secondary analysis, pairwise comparisons at each equivalent dose were performed followed by multiple comparison corrections, where appropriate. Where normality or equal variance of samples failed, nonparametric one-way ANOVA's (Kruskal-Wallis one-way ANOVA on ranks or Friedman repeated measures one-way ANOVA on ranks) were performed, followed by Dunn's correction. For assessment of the novelty-suppressed feeding results, Kaplan-Meier survival analysis was used followed by the Mantel-Cox log-rank test. The sample sizes, specific statistical tests used, and the main effects of our statistical analyses for each experiment are reported in Supplementary Information Table 1.

\section{Extended Data}

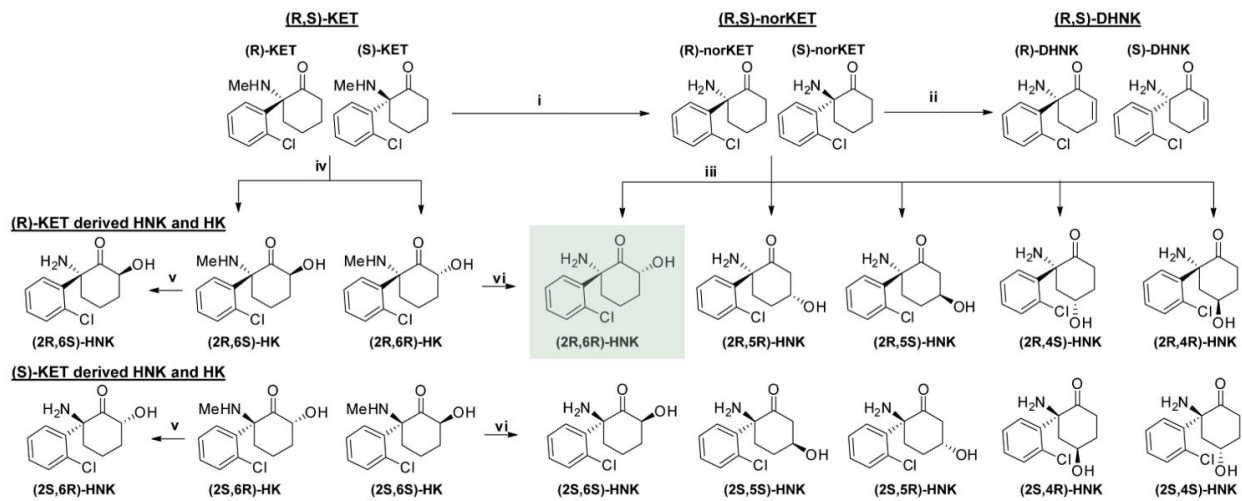

Extended Data Figure 1. Ketamine's metabolic transformations in vivo Ketamine is metabolised in vivo via P450 enzymatic transformations. (i) (R,S)-Ketamine (KET) is selectively demethylated to give (R,S)-norketamine (norKET). (ii) NorKET can be then dehydrogenated to give (R,S)-dehydronorketamine (DHNK). (iii) Alternatively, norKET can be hydroxylated to give the hydroxynorketamines (HNKs). (iv) (R,S)-KET can also be hydroxylated at the 6- position to give either the $E$-6-hydroxyketamine $((2 \mathrm{~S}, 6 \mathrm{R} ; 2 \mathrm{R}$, $6 \mathrm{~S})-\mathrm{HK})$ ) or Z-6-hydroxyketamine ((2S,6S;2R,6R)-HK)). (v) Demethylation of (2S,6R;2R, $6 \mathrm{~S})$-HK yields the production of (2S,6R;2R,6S)-hydroxynorketamine (HNK). (vi) Demethylation of (2S,6S;2R,6R)-HK further gives (2S,6S;2R,6R)-hydroxynorketamine (HNK). 

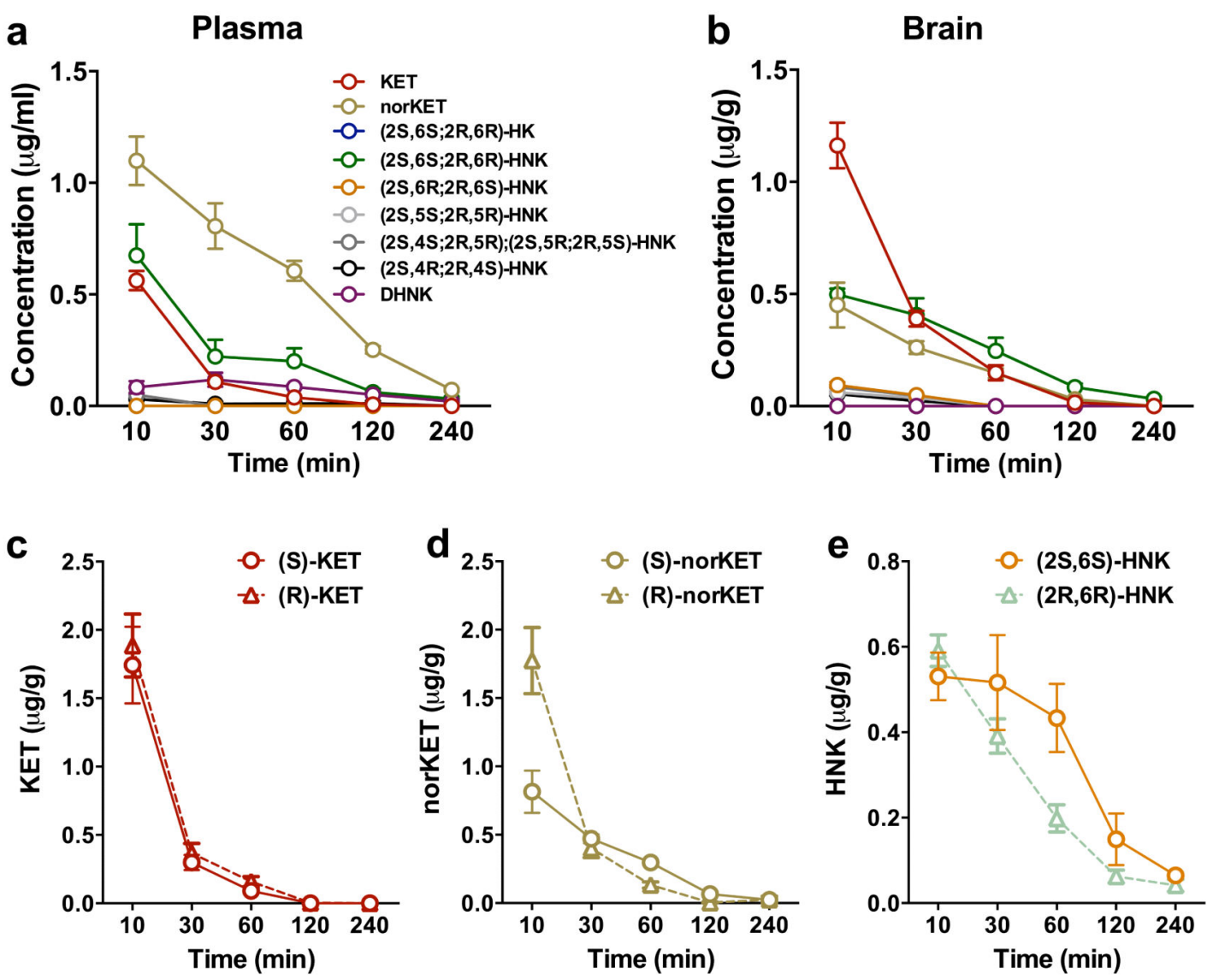

f $(R, S)-6,6$-dideuteroketamine
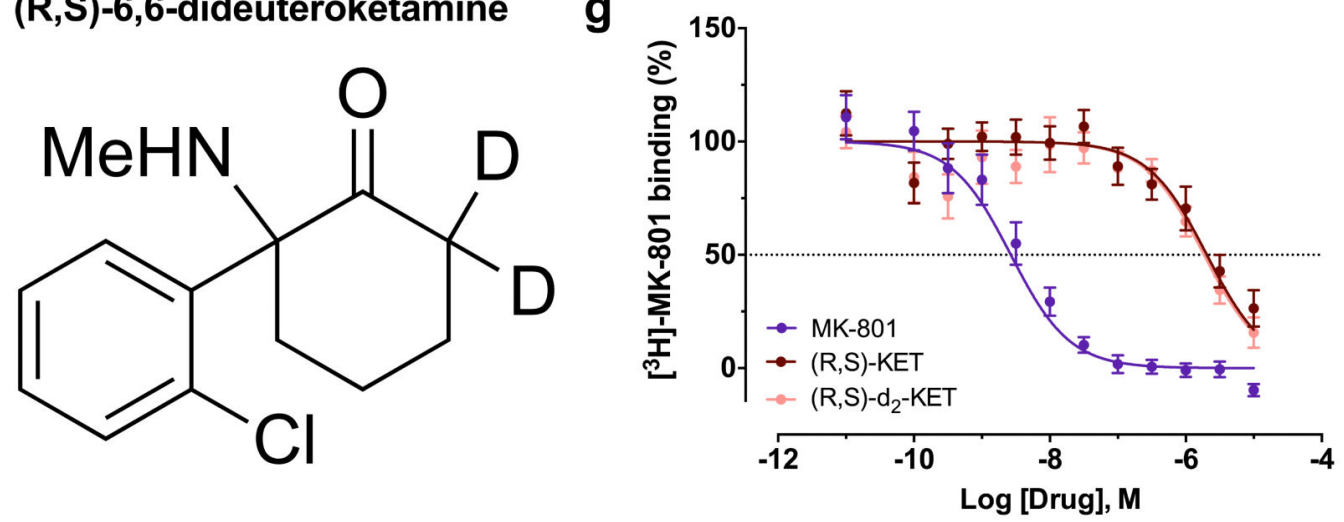

Extended Data Figure 2. Circulating levels of ketamine and its metabolites following i.p. administration in mice

a, Plasma and b, brain levels of ketamine (KET) and its metabolites following administration of (R,S)-KET (10 mg/kg) in mice. (c-d) Brain levels of c, KET, d, norketamine (norKET) and e, hydroxynorketamine (HNK) following administration of (S)and (R)-KET. f, Chemical structure of (R,S)-6,6-dideuteroketamine ((R,S)- $\left.\mathrm{d}_{2}-\mathrm{KET}\right)$, which g, displaces $\left[{ }^{3} \mathrm{H}\right]-\mathrm{MK}-801$ binding with a similar affinity to $(\mathrm{R}, \mathrm{S})-\mathrm{KET}((\mathrm{R}, \mathrm{S})-\mathrm{KET}$ : 
$\left.\mathrm{Ki}=799 \mathrm{nM}(\mathrm{R}, \mathrm{S})-\mathrm{d}_{2}-\mathrm{KET}: \mathrm{Ki}=883 \mathrm{nM}\right)$ (statistical analyses and $n$ numbers see

Supplementary Information Table 1).

a

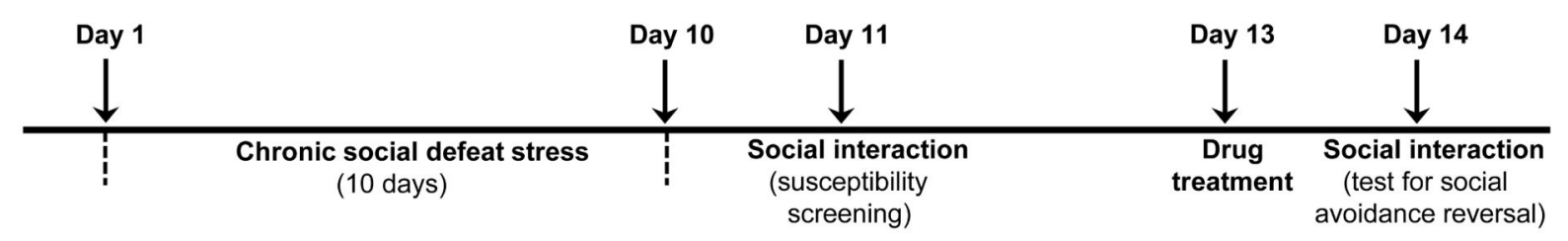

b

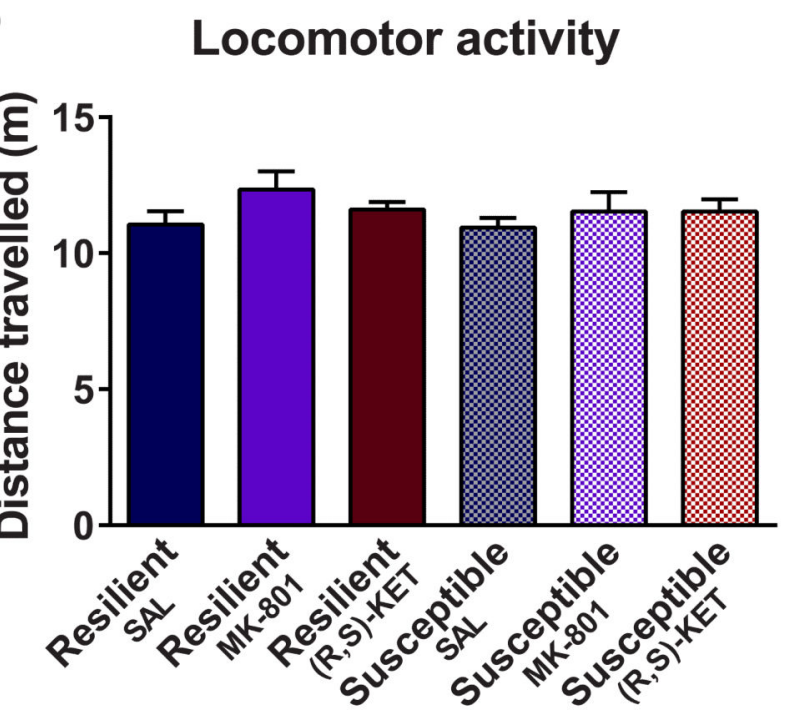

C Total compartmental crosses

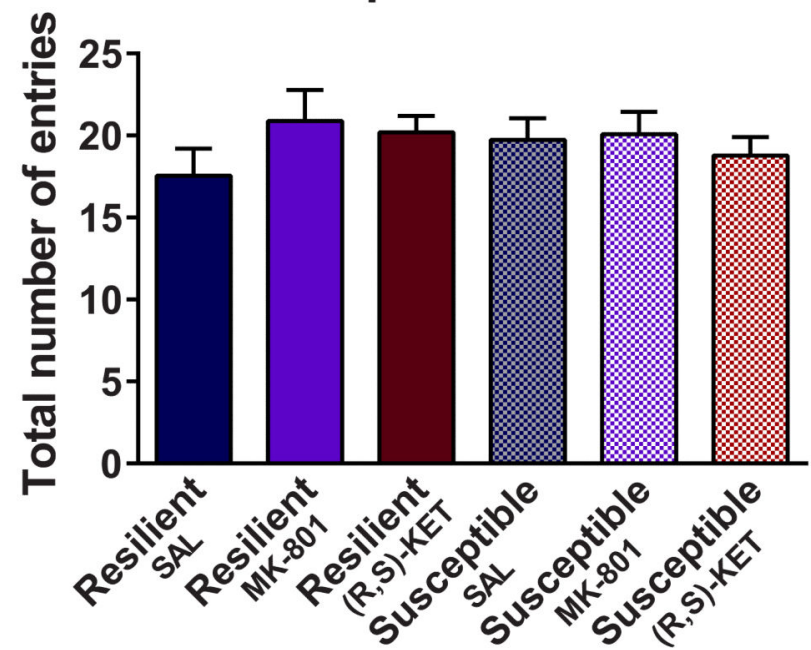

Extended Data Figure 3. Additional social defeat stress data

a, Chronic social defeat stress and social interaction/avoidance test timeline. (b-c),

Administration of (R,S)-ketamine (KET) or MK-801did not affect $\mathbf{b}$, locomotor activity or $\mathbf{c}$, total number of compartmental crosses in the social interaction apparatus. Data are means \pm S.E.M. $* * * p<0.001$. SAL, saline (statistical analyses and $n$ numbers see Supplementary Information Table 1). 
a

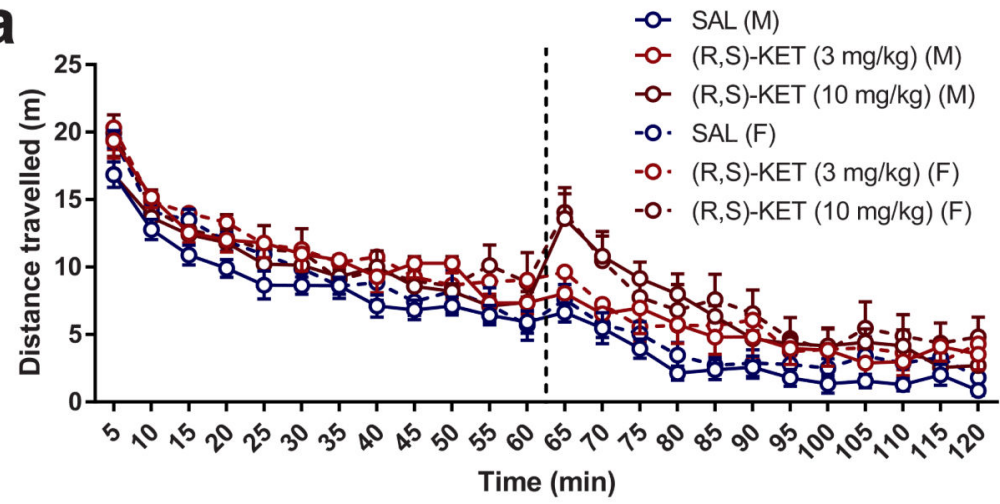

C

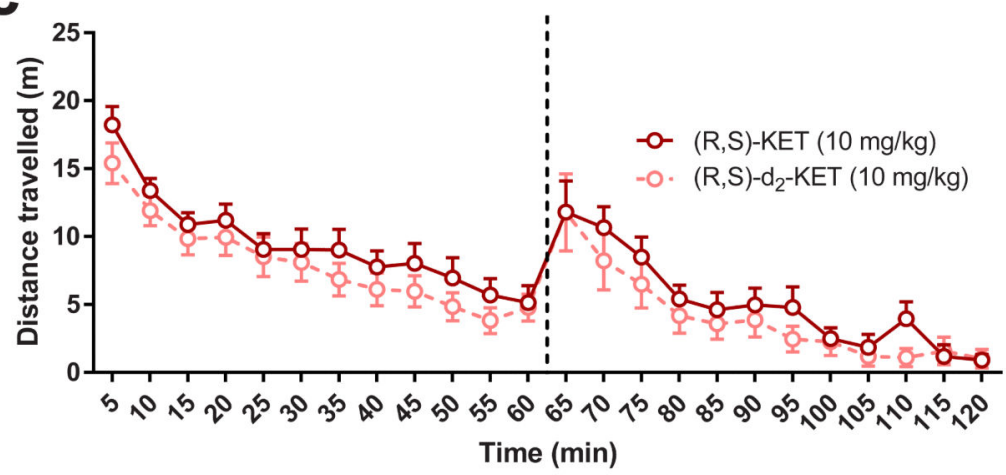

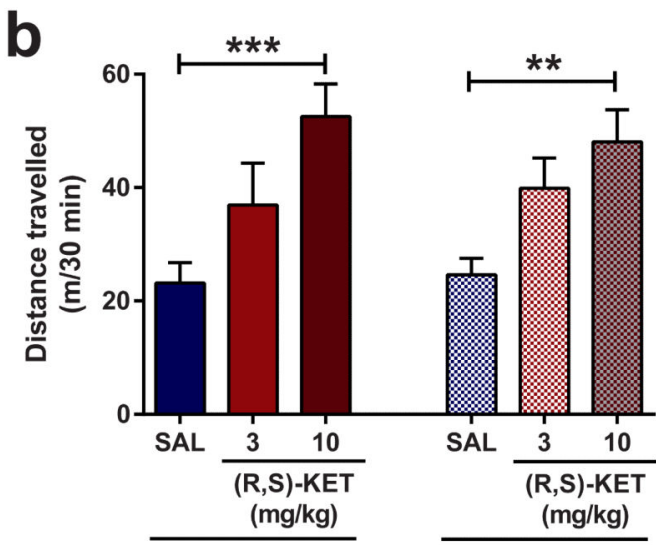

d

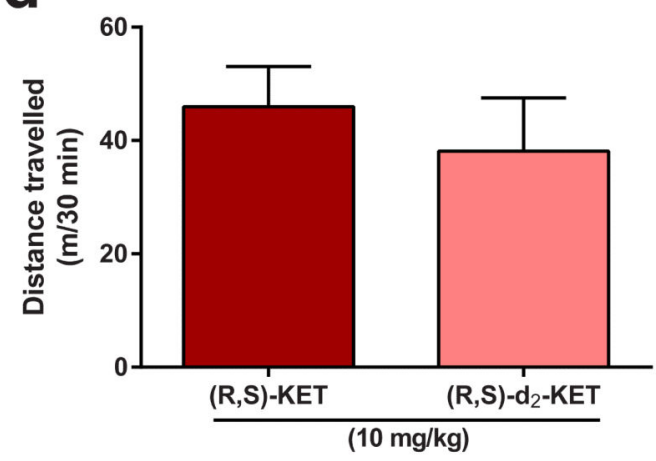

Extended Data Figure 4. Locomotor effects of (R,S)-ketamine and (R,S)-6,6-dideuteroketamine After recording baseline activity for $60 \mathrm{~min}$, mice received drug (marked by a vertical dashed line) and locomotor activity was monitored for another 1 hour. (a,b), Administration of (R,S)-KET (10 mg/kg), induced hyper-locomotor responses equally in both male and female mice. (c,d), (R,S)-ketamine (KET) and (R,S)-6,6-dideuteroketamine ((R,S)- $\left.\mathrm{d}_{2}-\mathrm{KET}\right)$ were equally potent in inducing a hyper-locomotor response at the dose of $10 \mathrm{mg} / \mathrm{kg}$. Data are means \pm S.E.M. ${ }^{*} p<0.05, * * p<0.01$. SAL, saline (statistical analyses and $n$ numbers see Supplementary Information Table 1). 
Learned helplessness
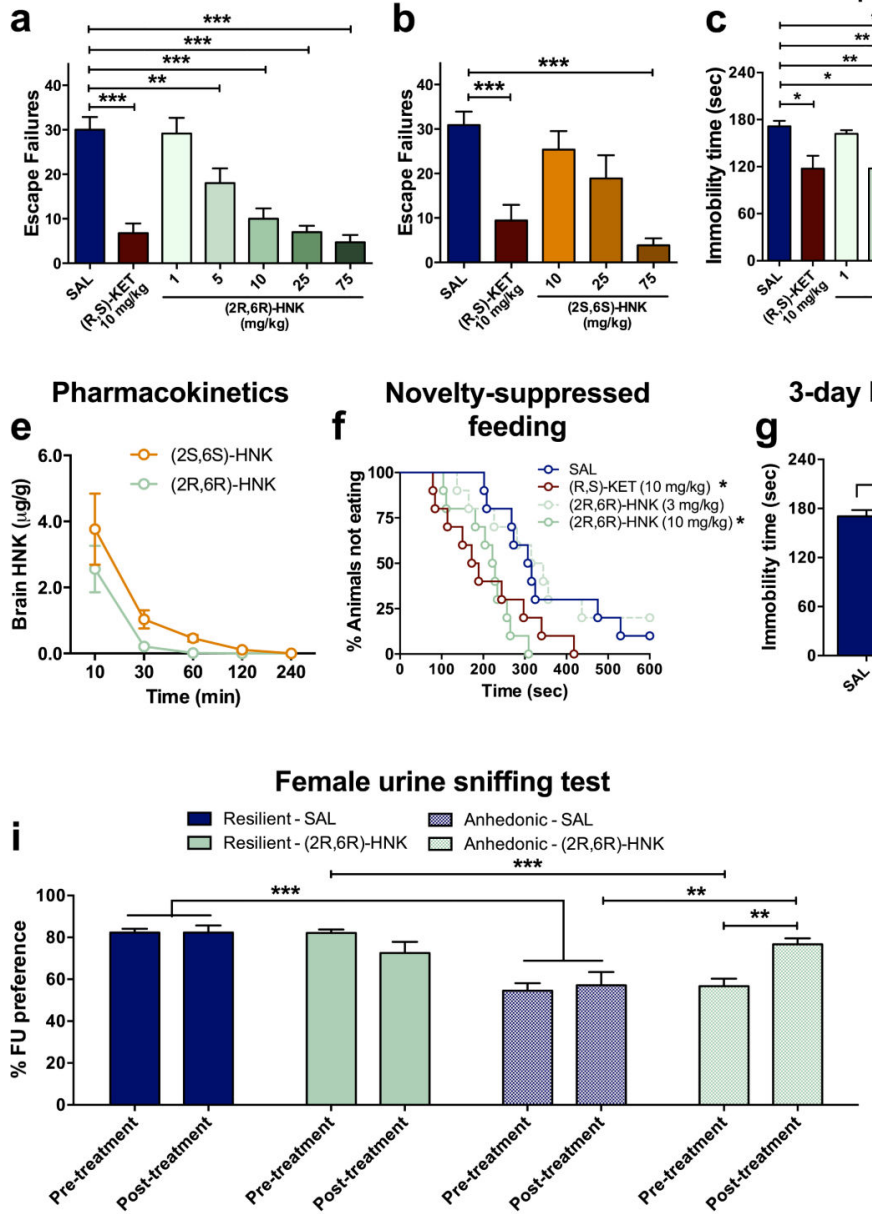

Female urine sniffing test 6R)- and (2S,6S)-hydroxynorketamine
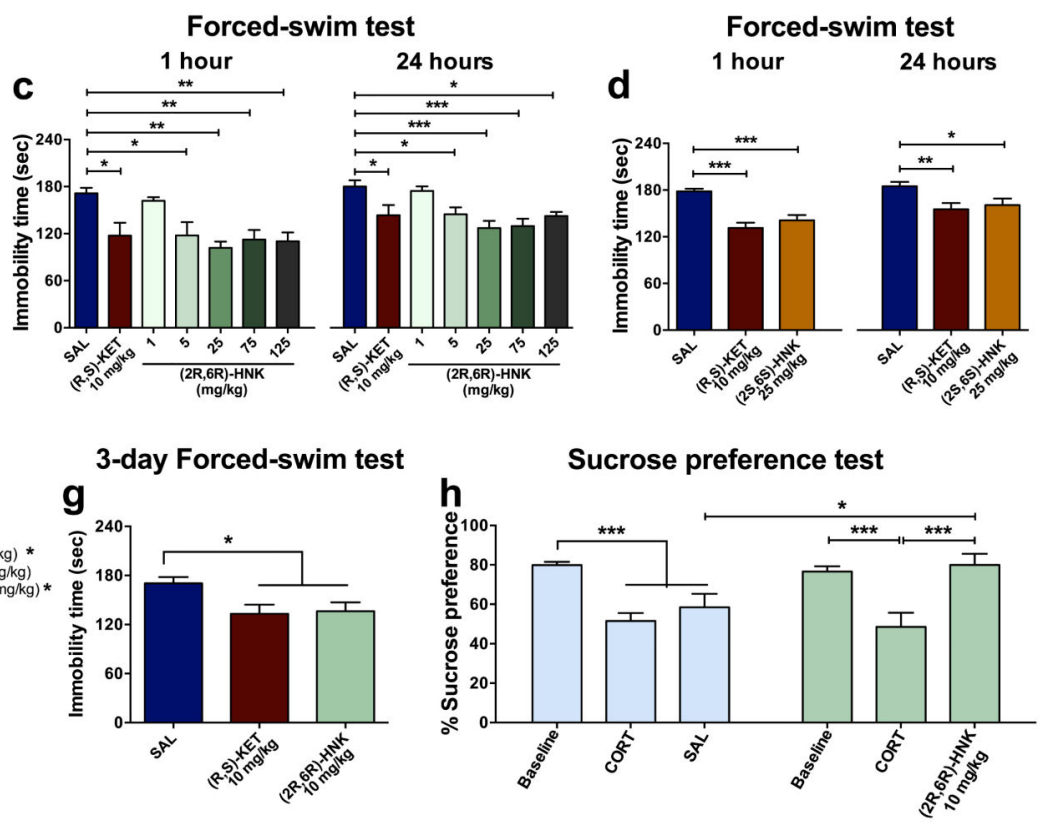

Social defeats

j

Locomotor activity

K Total compartmental crosses
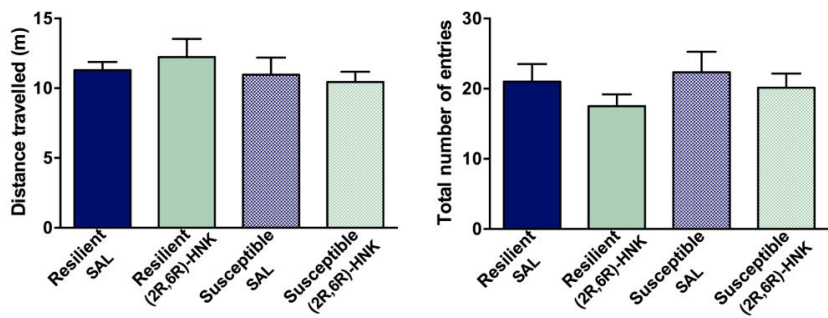

Extended Data Figure 5. Acute and sustained antidepressant and anti-anhedonic effects of (2R,

a, A single injection of (2R,6R)-HNK resulted in dose-dependent antidepressant-like responses in the learned helplessness test at the doses of $5-75 \mathrm{mg} / \mathrm{kg}$. b, A single injection of (2S,6S)-hydroxynorketamine (HNK) induced antidepressant-like effects in the learned helplessness test at the dose of $75 \mathrm{mg} / \mathrm{kg}$. c, Administration of (2R,6R)-HNK induced dosedependent antidepressant effects in the 1- and 24-hour forced-swim test. d, Administration of $(2 \mathrm{~S}, 6 \mathrm{~S})-\mathrm{HNK}$ at the dose of $25 \mathrm{mg} / \mathrm{kg}$ induced antidepressant effects in the 1- and 24hour forced-swim test. e, Despite the greater antidepressant efficacy of (2R,6R)-HNK, administration of $(2 \mathrm{~S}, 6 \mathrm{~S})-\mathrm{HNK}(\mathrm{HNK})$ results in higher brain hydroxynorketamine levels compared to (2R,6R)-HNK. f, (2R,6R)-HNK manifested dose-dependent antidepressant-like effects in the novelty-suppressed feeding test. $\mathbf{g}$, Similar to (R,S)-ketamine (KET), the antidepressant-like effects of (2R,6R)-HNK in the forced-swim test persisted for at least 3 days post-treatment. $\mathbf{h}$, A single administration of (2R,6R)-HNK reversed chronic corticosterone-induced decreases in sucrose preference. i, A single administration of (2R, 6R)-HNK reversed chronic corticosterone-induced decrease in female urine sniffing preference, specifically in mice that developed an anhedonic phenotype. Administration of (2R,6R)-HNK was not associated with changes in $\mathbf{j}$, locomotor activity or $\mathbf{k}$, total 
compartmental crosses in the social interaction test following chronic social defeat stress. Data are means \pm S.E.M. $* p<0.05, * * p<0.01, * * * p<0.001$. SAL, saline (statistical analyses and $n$ numbers see Supplementary Information Table 1).
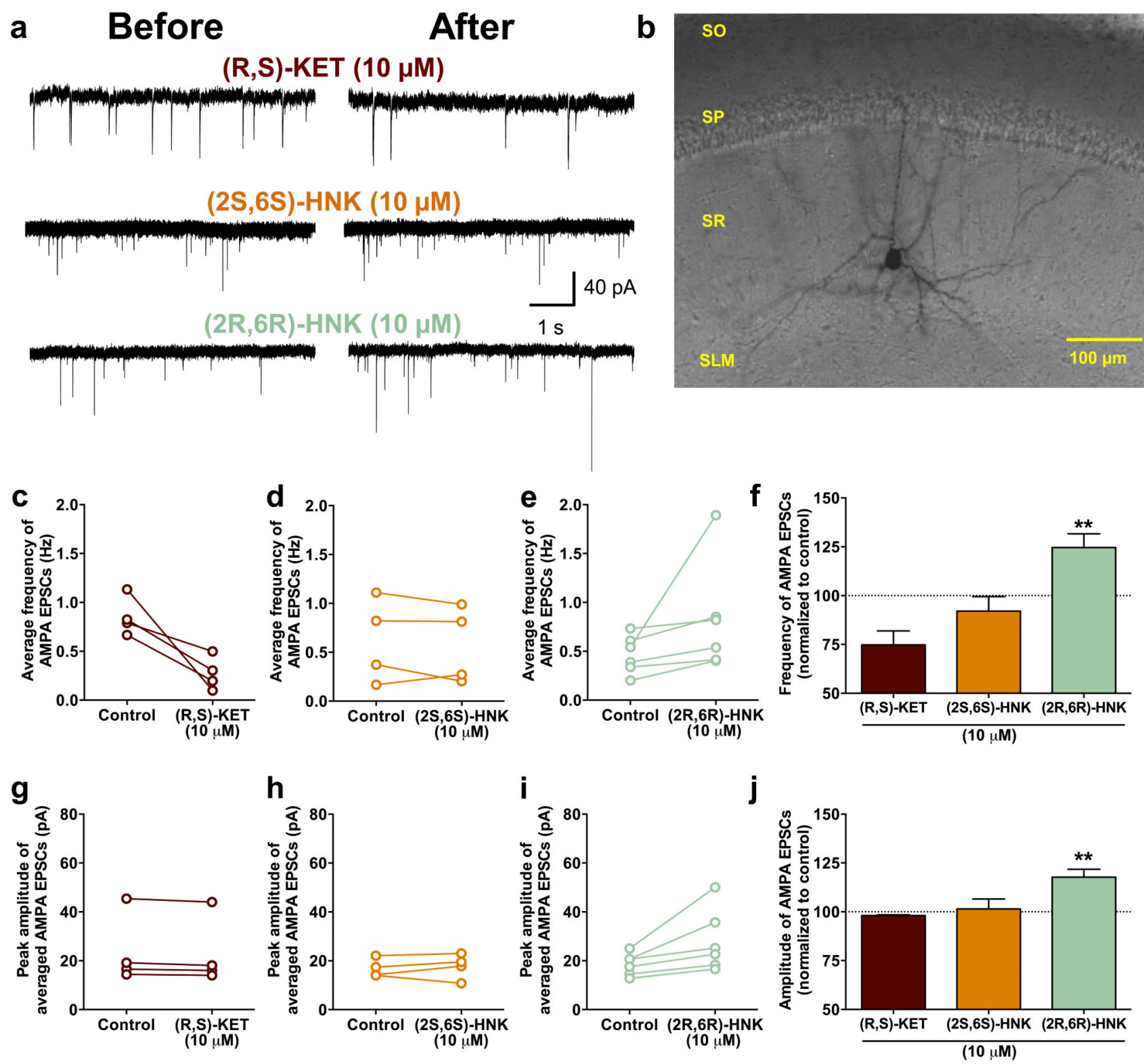

Extended Data Figure 6. (2R,6R)-hydroxynorketamine rapidly increases the frequency and amplitude of AMPA receptor spontaneous excitatory postsynaptic currents in the hippocampus a, Representative traces of spontaneous excitatory postsynaptic currents (sEPSCs) mediated via AMPA receptors during baseline (before) and 20 min following drug administration (after) in b, example CA1 stratum radiatum interneuron recorded from a rat hippocampal slice. (c-j) Twenty-min exposure of (e,i), (2R,6R)-hydroxynorketamine (HNK), but not (c,g), $(\mathrm{R}, \mathrm{S})$-ketamine $(\mathrm{KET})$ or $(\mathbf{d}, \mathbf{h}),(2 \mathrm{~S}, 6 \mathrm{~S})$-HNK increased AMPA sEPSCs frequency and amplitude compared to baseline. Data are means \pm S.E.M. ${ }^{*} p<0.05, * * p<0.01, * * * p<0.001$ 
(statistical analyses and $n$ numbers see Supplementary Information Table 1). Abbreviations: FST, forced-swim test; NBQX, 2,3-dihydroxy-6-nitro-7-sulfamoyl-benzo[f]quinoxaline-2,3dione; SAL, saline; SLM, stratum lacunosum-moleculare; SO, stratum oriens; SP, stratum pyramidale; SR, stratum radiatum.

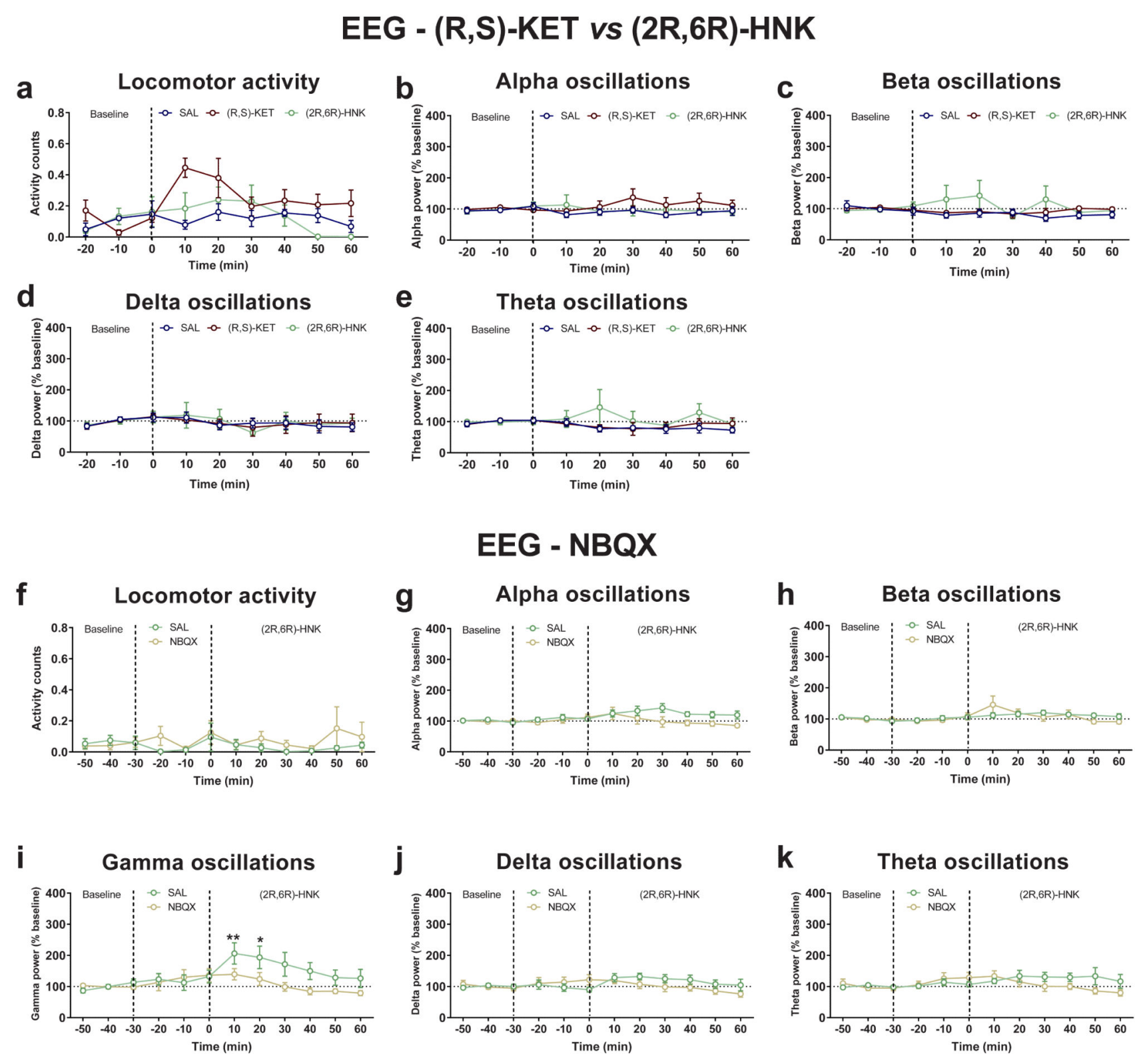

Extended Data Figure 7. Administration of the AMPA receptor antagonist, NBQX, prevents (2R, 6R)-HNK-induced increases in gamma oscillations in vivo

a, Administration of (R,S)-ketamine (KET), but not (2R,6R)-hydroxynorketamine (HNK), increased locomotor home-cage activity of mice. Neither (R,S)-KET, nor (2R,6R)-HNK altered cortical b, alpha, $\mathbf{c}$, beta, $\mathbf{d}$, delta or $\mathbf{e}$, theta oscillations in vivo. (f-k) Pre-treatment with the AMPA receptor antagonist, NBQX, did not change the $\mathbf{f}$, locomotor activity, $\mathbf{g}$, alpha, $\mathbf{h}$, beta, $\mathbf{j}$, delta or $\mathbf{k}$, theta oscillations, but it $\mathbf{i}$, prevented (2R,6R)-HNK-induced 
increases of gamma oscillations in vivo. Data are means \pm S.E.M. ${ }^{*} p<0.05, * * p<0.01$ (statistical analyses and $n$ numbers see Supplementary Information Table 1). Abbreviations: NBQX, 2,3-dihydroxy-6-nitro-7-sulfamoyl-benzo[f]quinoxaline-2,3-dione; SAL, saline.

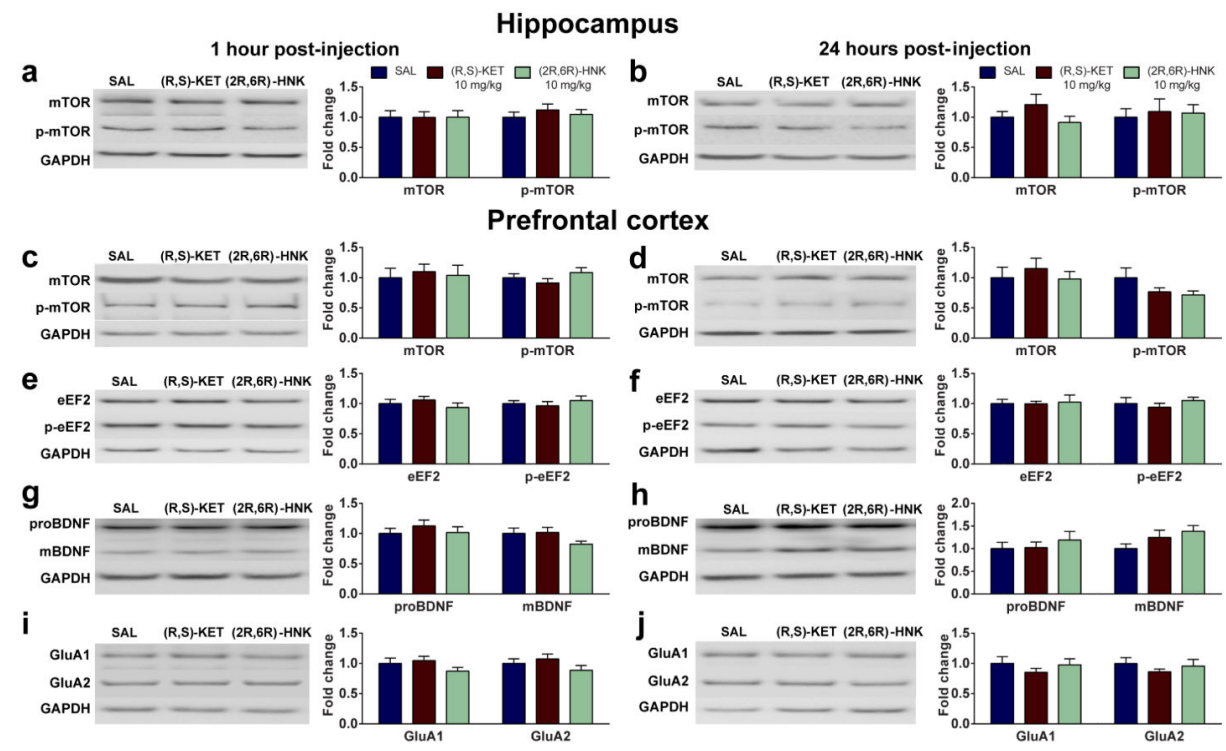

Extended Data Figure 8. Effects of (2R,6R)-hydroxynorketamine on synaptoneurosome protein and protein phosphorylation levels

A single administration of (R,S)-ketamine (KET, $10 \mathrm{mg} / \mathrm{kg}$ ) or (2R,6R)-hydroxynorketamine $(\mathrm{HNK}, 10 \mathrm{mg} / \mathrm{kg}$ ) (a,b), did not alter levels of mTOR or phosphorylated mTOR 1- or 24hours post-injection in the hippocampus of mice. (b-i), Administration of (R,S)-KET or (2R, 6R)-HNK did not alter levels of (c,d), mTOR/phosphorylated mTOR, (e,f), eEF2/ phosphorylated eEF2, (g,h), proBDNF/mBDNF, or (i,j), GluA1/GluA2 in the prefrontal cortex of mice. The values for the phosphorylated forms of proteins were normalised to phosphorylation-independent levels of the same protein. Phosphorylation-independent levels of proteins were normalised to GAPDH. Data are means \pm S.E.M, and was normalised to the saline-treated control group for each protein. Images are cropped; see Supplementary Fig. 1 for complete blot images * $p<0.05$ (statistical analyses and $n$ numbers see Supplementary Information Table 1). Abbreviations: eEF2, eukaryotic translation elongation factor 2; GAPDH, Glyceraldehyde 3-phosphate dehydrogenase; mBDNF, mature brain-derived neurotrophic factor; mTOR, mammalian target of rapamycin; proBDNF, pro-brain-derived neurotrophic factor; SAL, saline. 


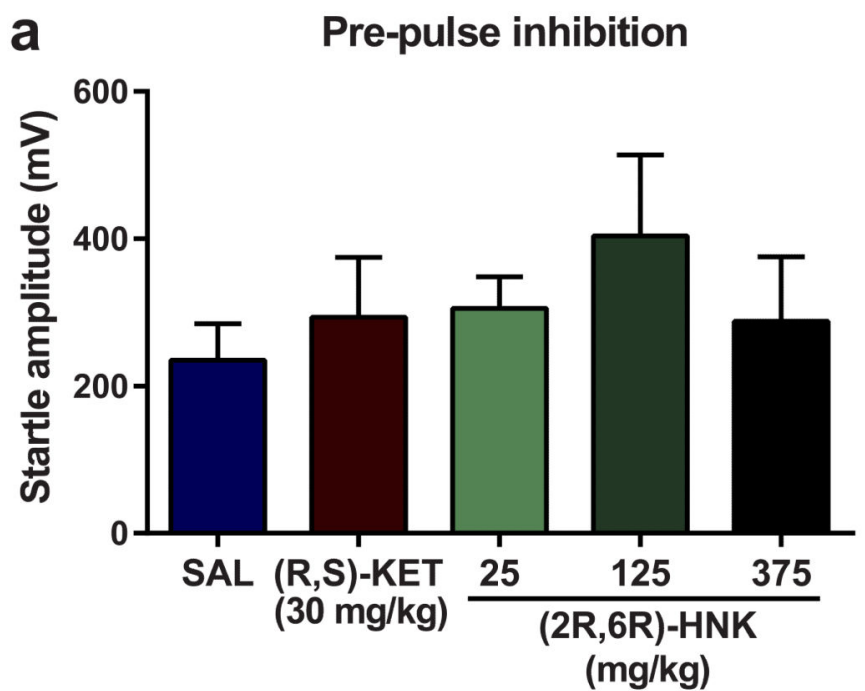

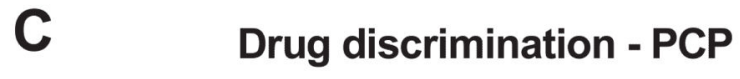

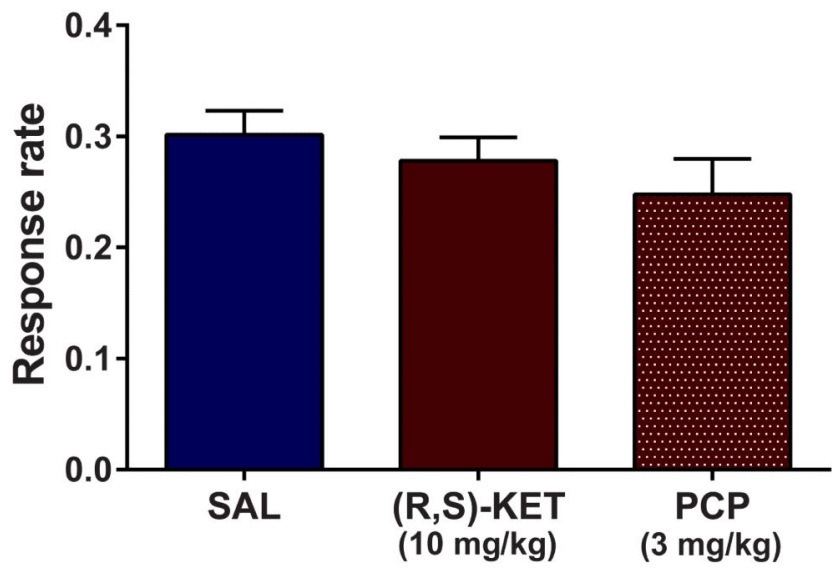

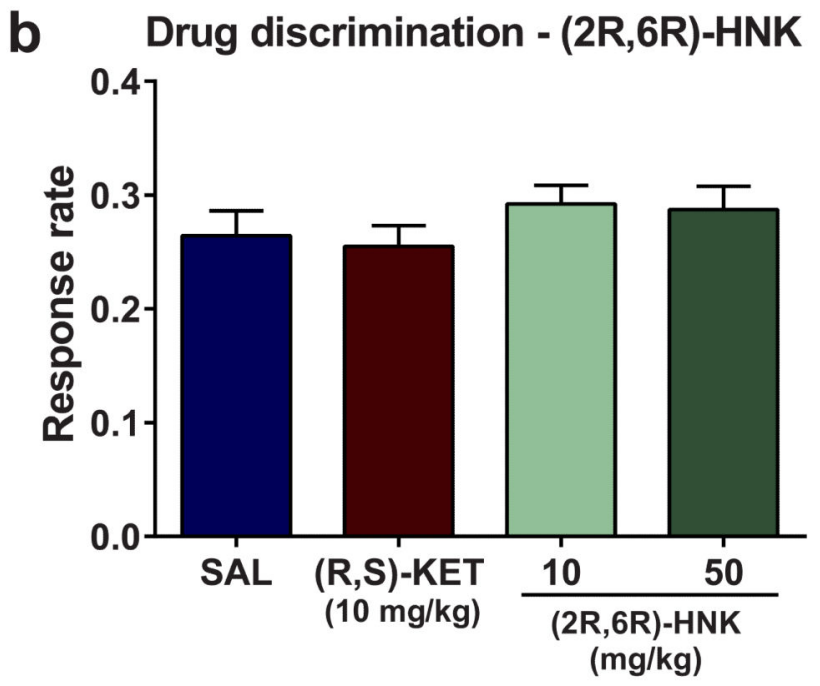

d

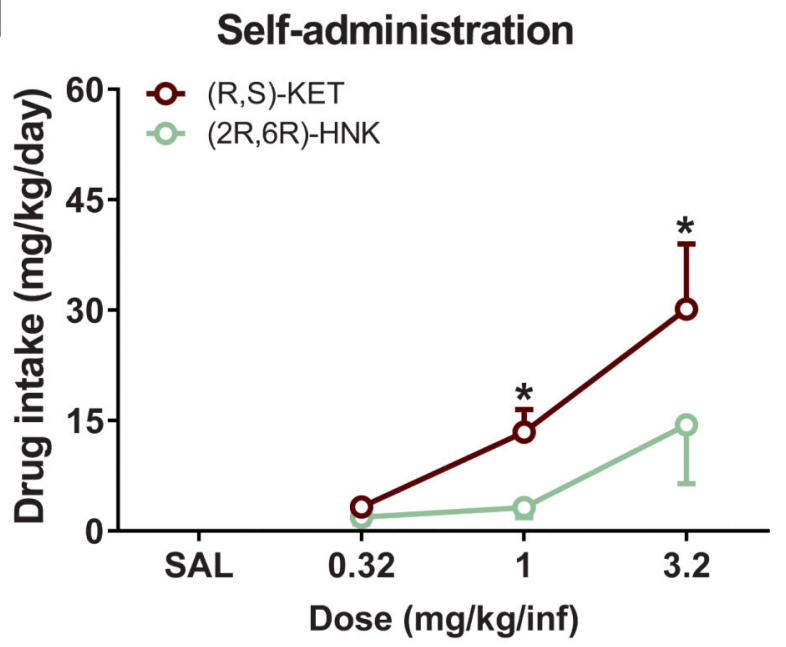

Extended Data Figure 9. (2R,6R)-hydroxynorketamine administration does not alter startle amplitude, self-administration drug intake or drug discrimination response rate

a, Startle amplitude as measured in the pre-pulse inhibition task was not affected by administration of (R,S)-ketamine (KET) or (2R,6R)-hydroxynorketamine (HNK). (b,c),

Response rate of overall lever pressing per sec in the drug discrimination paradigm was not changed by administration of $\mathbf{b},(\mathrm{R}, \mathrm{S})-\mathrm{KET},(2 \mathrm{R}, 6 \mathrm{R})-\mathrm{HNK}$ or $\mathbf{c}$, phencyclidine (PCP). d, Unlike ketamine, (2R,6R)-HNK did not alter drug intake in the self-administration task in mice. Data are means \pm S.E.M. $* p<0.05$ (statistical analyses and $n$ numbers see Supplementary Information Table 1). SAL, saline.

\section{Supplementary Material}

Refer to Web version on PubMed Central for supplementary material. 


\section{Acknowledgements}

We thank Asaf Keller (University of Maryland, Baltimore) for his assistance with the EEG experiments, Mary Kay Lobo (University of Maryland, Baltimore) for assistance with the social defeat experiments, Bhagavathy Alkondon (University of Maryland, Baltimore) for the rat hippocampal slice preparation and biocytin processing, Victoria Meadows and Sam Krimmel (both University of Maryland, Baltimore) for assistance with behavioural experiments and manuscript review, Edna Pereira (University of Maryland, Baltimore) for critical comments on the manuscript and Figures, David Luckenbaugh (NIMH Intramural Research Program) for assistance with statistical analysis, and Curtis Moore (University of California, San Diego) for the small molecule X-ray crystallography. Research was supported by NIMH grants MH099345 and MH107615 to TDG and MH086828 to SMT, and the NIA (RM, IWW), NIMH (CAZ), and NCATS (CJT) NIH intramural research programs. Receptor binding profiles, $\mathrm{K}_{\mathrm{i}}$ determinations, and functional assays were supported by the NIMH Psychoactive Drug Screening Program, Contract \# HHSN-271-2008-025C, to Bryan L. Roth in conjunction with Ms. Jamie Driscoll (NIMH, Bethesda, MD, USA). Initial synthesis of the ketamine metabolites used in this study was supported by NIA Contract \#HHSN271201000008I to IWW.

\section{References}

1. Kessler RC, et al. The epidemiology of major depressive disorder: results from the National Comorbidity Survey Replication (NCS-R). JAMA. 2003; 289:3095-3105. [PubMed: 12813115]

2. Trivedi MH, et al. Evaluation of outcomes with citalopram for depression using measurement-based care in STAR*D: implications for clinical practice. Am J Psychiatry. 2006; 163:28-40. [PubMed: 16390886]

3. Rush AJ, et al. Acute and longer-term outcomes in depressed outpatients requiring one or several treatment steps: a STAR*D report. Am J Psychiatry. 2006; 163:1905-1917. [PubMed: 17074942]

4. Zarate CA Jr. et al. A randomized trial of an N-methyl-D-aspartate antagonist in treatment-resistant major depression. Archives of general psychiatry. 2006; 63:856-864. [PubMed: 16894061]

5. Berman RM, et al. Antidepressant effects of ketamine in depressed patients. Biol Psychiatry. 2000; 47:351-354. [PubMed: 10686270]

6. Diazgranados N, et al. A randomized add-on trial of an N-methyl-D-aspartate antagonist in treatment-resistant bipolar depression. Archives of general psychiatry. 2010; 67:793-802. [PubMed: 20679587]

7. Lally N, et al. Anti-anhedonic effect of ketamine and its neural correlates in treatment-resistant bipolar depression. Transl Psychiatry. 2014; 4:e469. [PubMed: 25313512]

8. Murrough JW, et al. Antidepressant efficacy of ketamine in treatment-resistant major depression: a two-site randomized controlled trial. Am J Psychiatry. 2013; 170:1134-1142. [PubMed: 23982301]

9. Morgan CJ, Curran HV. Ketamine use: a review. Addiction. 2012; 107:27-38. [PubMed: 21777321]

10. Newport DJ, et al. Ketamine and Other NMDA Antagonists: Early Clinical Trials and Possible Mechanisms in Depression. American Journal of Psychiatry. 2015; 172:950-966. [PubMed: 26423481]

11. Leung LY, Baillie TA. Comparative pharmacology in the rat of ketamine and its two principal metabolites, norketamine and (Z)-6-hydroxynorketamine. J Med Chem. 1986; 29:2396-2399. [PubMed: 3783598]

12. Moaddel R, et al. Sub-anesthetic concentrations of (R,S)-ketamine metabolites inhibit acetylcholine-evoked currents in alpha7 nicotinic acetylcholine receptors. Eur J Pharmacol. 2013; 698:228-234. [PubMed: 23183107]

13. Ebert B, Mikkelsen S, Thorkildsen C, Borgbjerg FM. Norketamine, the main metabolite of ketamine, is a non-competitive NMDA receptor antagonist in the rat cortex and spinal cord. Eur J Pharmacol. 1997; 333:99-104. [PubMed: 9311667]

14. Yang C, et al. R-ketamine: a rapid-onset and sustained antidepressant without psychotomimetic side effects. Transl Psychiatry. 2015; 5:e632. [PubMed: 26327690]

15. Maeng S, et al. Cellular mechanisms underlying the antidepressant effects of ketamine: role of alpha-amino-3-hydroxy-5-methylisoxazole-4-propionic acid receptors. Biol Psychiatry. 2008; 63:349-352. [PubMed: 17643398]

16. Autry AE, et al. NMDA receptor blockade at rest triggers rapid behavioural antidepressant responses. Nature. 2011; 475:91-95. [PubMed: 21677641] 
17. Desta Z, et al. Stereoselective and regiospecific hydroxylation of ketamine and norketamine. Xenobiotica. 2012; 42:1076-1087. [PubMed: 22612619]

18. Adams JD Jr. Baillie TA, Trevor AJ, Castagnoli N Jr. Studies on the biotransformation of ketamine. 1-Identification of metabolites produced in vitro from rat liver microsomal preparations. Biomed Mass Spectrom. 1981; 8:527-538. [PubMed: 7317567]

19. Zarate CA Jr. et al. Relationship of ketamine's plasma metabolites with response, diagnosis, and side effects in major depression. Biol Psychiatry. 2012; 72:331-338. [PubMed: 22516044]

20. Carrier N, Kabbaj M. Sex differences in the antidepressant-like effects of ketamine. Neuropharmacology. 2013; 70:27-34. [PubMed: 23337256]

21. Franceschelli A, Sens J, Herchick S, Thelen C, Pitychoutis PM. Sex differences in the rapid and the sustained antidepressant-like effects of ketamine in stress-naive and "depressed" mice exposed to chronic mild stress. Neuroscience. 2015; 290:49-60. [PubMed: 25595985]

22. Irifune M, Shimizu T, Nomoto M, Fukuda T. Involvement of N-methyl-D-aspartate (NMDA) receptors in noncompetitive NMDA receptor antagonist-induced hyperlocomotion in mice. Pharmacol Biochem Behav. 1995; 51:291-296. [PubMed: 7667342]

23. Gant TG. Using deuterium in drug discovery: leaving the label in the drug. J Med Chem. 2014; 57:3595-3611. [PubMed: 24294889]

24. Duman RS, Aghajanian GK, Sanacora G, Krystal JH. Synaptic plasticity and depression: new insights from stress and rapid-acting antidepressants. Nat Med. 2016; 22:238-249. [PubMed: 26937618]

25. Li N, et al. mTOR-dependent synapse formation underlies the rapid antidepressant effects of NMDA antagonists. Science. 2010; 329:959-964. [PubMed: 20724638]

26. Koike H, Iijima M, Chaki S. Involvement of AMPA receptor in both the rapid and sustained antidepressant-like effects of ketamine in animal models of depression. Behav Brain Res. 2011; 224:107-111. [PubMed: 21669235]

27. Koike H, Chaki S. Requirement of AMPA receptor stimulation for the sustained antidepressant activity of ketamine and LY341495 during the forced swim test in rats. Behav Brain Res. 2014 271:111-115. [PubMed: 24909673]

28. Whittington MA, Traub RD, Kopell N, Ermentrout B, Buhl EH. Inhibition-based rhythms: experimental and mathematical observations on network dynamics. Int J Psychophysiol. 2000; 38:315-336. [PubMed: 11102670]

29. Cunningham MO, Davies CH, Buhl EH, Kopell N, Whittington MA. Gamma oscillations induced by kainate receptor activation in the entorhinal cortex in vitro. J Neurosci. 2003; 23:9761-9769. [PubMed: 14586003]

30. Muthukumaraswamy SD, et al. Evidence that Subanesthetic Doses of Ketamine Cause Sustained Disruptions of NMDA and AMPA-Mediated Frontoparietal Connectivity in Humans. J Neurosci. 2015; 35:11694-11706. [PubMed: 26290246]

31. Lepack AE, Fuchikami M, Dwyer JM, Banasr M, Duman RS. BDNF release is required for the behavioral actions of ketamine. Int J Neuropsychopharmacol. 2015:18.

32. De Vry J, Jentzsch KR. Role of the NMDA receptor NR2B subunit in the discriminative stimulus effects of ketamine. Behav Pharmacol. 2003; 14:229-235. [PubMed: 12799525]

33. Moaddel R, et al. The distribution and clearance of (2S,6S)-hydroxynorketamine, an active ketamine metabolite, in Wistar rats. Pharmacol Res Perspect. 2015; 3:e00157. [PubMed: 26171236]

\section{References (Methods)}

34. Donahue RJ, Muschamp JW, Russo SJ, Nestler EJ, Carlezon WA Jr. Effects of striatal DeltaFosB overexpression and ketamine on social defeat stress-induced anhedonia in mice. Biol Psychiatry. 2014; 76:550-558. [PubMed: 24495460]

35. Malkesman $\mathrm{O}$, et al. The female urine sniffing test: a novel approach for assessing reward-seeking behavior in rodents. Biol Psychiatry. 2010; 67:864-871. [PubMed: 20034613] 
36. Zanos P, et al. The Prodrug 4-Chlorokynurenine Causes Ketamine-Like Antidepressant Effects, but Not Side Effects, by NMDA/GlycineB-Site Inhibition. J Pharmacol Exp Ther. 2015; 355:76-85. [PubMed: 26265321]

37. Chiu J, et al. Chronic ethanol exposure alters MK-801 binding sites in the cerebral cortex of the near-term fetal guinea pig. Alcohol. 1999; 17:215-221. [PubMed: 10231169]

38. Raver SM, Haughwout SP, Keller A. Adolescent cannabinoid exposure permanently suppresses cortical oscillations in adult mice. Neuropsychopharmacology. 2013; 38:2338-2347. [PubMed: 23822952]

39. Bokil H, Andrews P, Kulkarni JE, Mehta S, Mitra PP. Chronux: a platform for analyzing neural signals. J Neurosci Methods. 2010; 192:146-151. [PubMed: 20637804] 
a

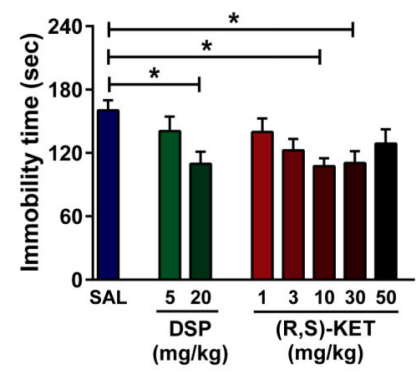

C

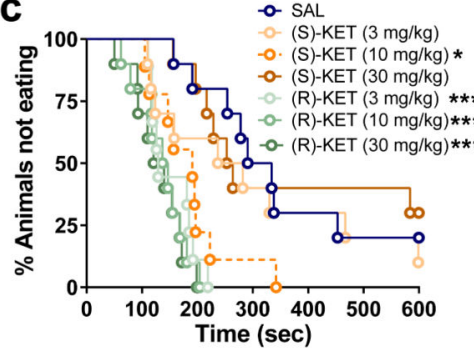

f
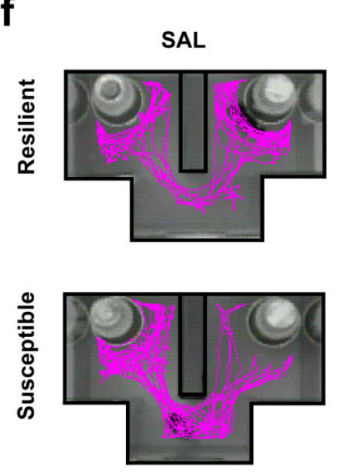

24 hours post-injection

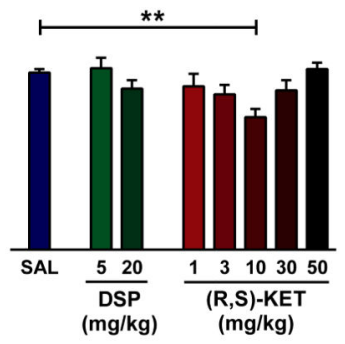

d

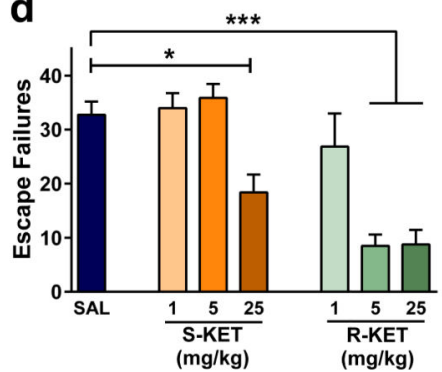

e 1 hour post-injection

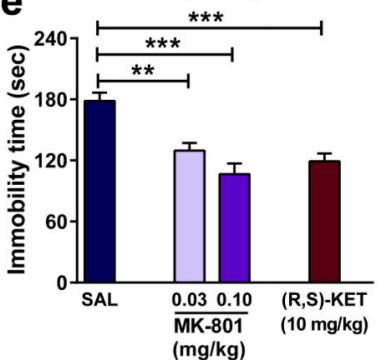

g

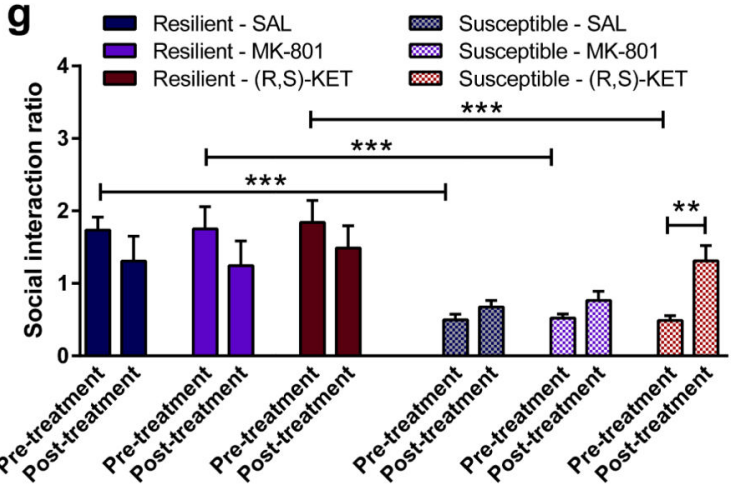

Figure 1. NMDA receptor inhibition does not explain the antidepressant actions of ketamine Antidepressant-like responses of a, (R,S)-ketamine (KET) and desipramine (DSP) in the forced-swim test 1- and 24-hours post-treatment. (b-d) Compared to (S)-KET, (R)-KET showed greater and longer-lasting antidepressant-like effects in the $\mathbf{b}$, forced-swim test, $\mathbf{c}$, novelty-suppressed feeding and $\mathbf{d}$, learned helplessness paradigms. (e-g), The alternative NMDAR antagonist MK-801 did not elicit e, 24-hour antidepressant actions in the forcedswim test and (f,g) did not reverse social avoidance induced by chronic social defeat stress. Data are means \pm S.E.M. ${ }^{*} p<0.05, * * p<0.01, * * * p<0.001$ (statistical analyses and $n$ numbers see Supplementary Information Table 1). 


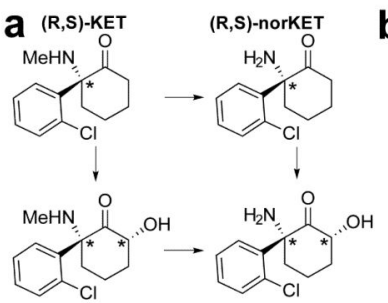

$(2 S, 6 S ; 2 R, 6 R)-H K \quad(2 S, 6 S ; 2 R, 6 R)-H N K$
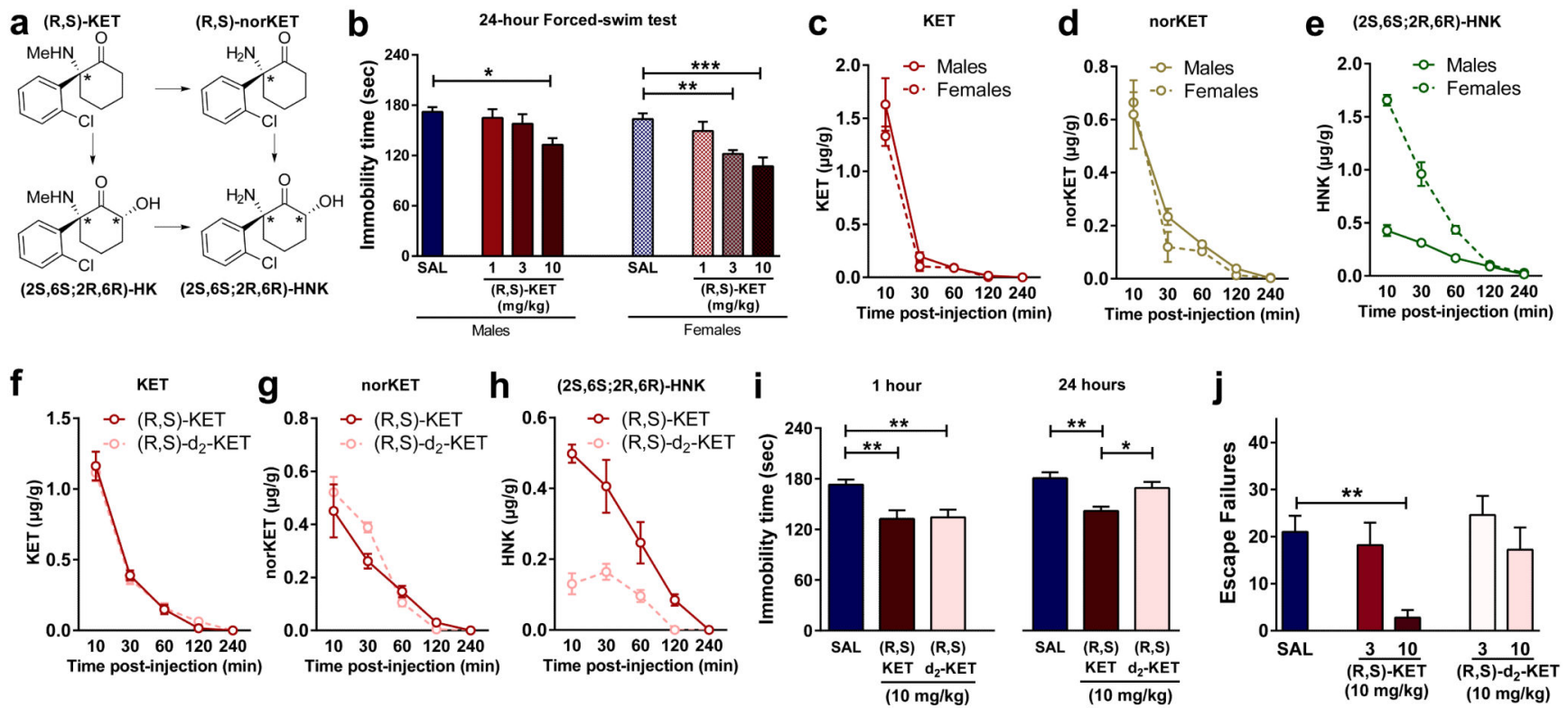

k

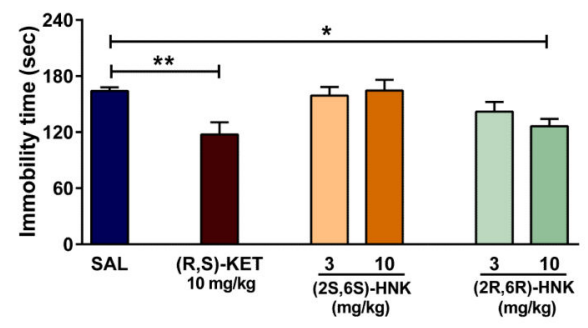

I

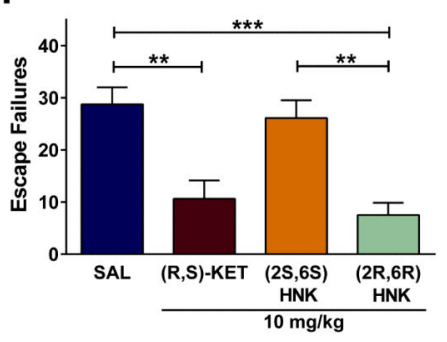

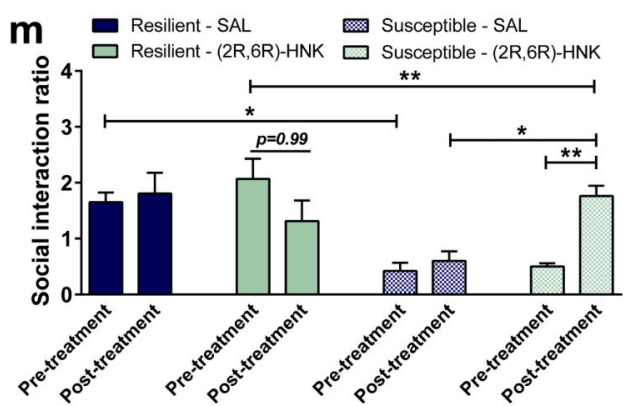

Figure 2. Metabolism of ketamine to (2R,6R)-HNK is necessary and sufficient to exert ketaminerelated antidepressant actions

a, Simplified diagram of (R,S)-KET's metabolism. b, Greater antidepressant-like actions of ketamine in female mice compared to males are associated with (c-e), higher brain levels of e, $(2 \mathrm{~S}, 6 \mathrm{~S} ; 2 \mathrm{R}, 6 \mathrm{R})$-hydroxynorketamine (HNK), but not $\mathbf{c}$, KET, or d, nor-KET. (f-h), Brain levels of $\mathbf{f}, \mathrm{KET}, \mathbf{g}$, nor-KET and $\mathbf{h},(2 \mathrm{~S}, 6 \mathrm{~S} ; 2 \mathrm{R}, 6 \mathrm{R})$-HNK following administration of (R,S)KET and 6,6-dideuteroketamine ((R,S)- $\left.\mathrm{d}_{2}-\mathrm{KET}\right)$. (i-j), Effects of (R,S)-KET and (R,S)- $\mathrm{d}_{2}-$ KET in the i, 1- and 24-hours forced-swim and the $\mathbf{j}$, learned helplessness tests. (k-l), Compared to (2S,6S)-HNK, (2R,6R)-HNK manifested greater potency and longer-lasting antidepressant-like effects in the $\mathbf{k}$, forced-swim test and $\mathbf{l}$, learned helplessness paradigms. $\mathbf{m},(2 \mathrm{R}, 6 \mathrm{R})-\mathrm{HNK}$ reversed chronic social defeat-induced social interaction deficits. Data are means \pm S.E.M. $* p<0.05$, $* * p<0.01$, $* * * p<0.001$ (statistical analyses and $n$ numbers see Supplementary Information Table 1). 


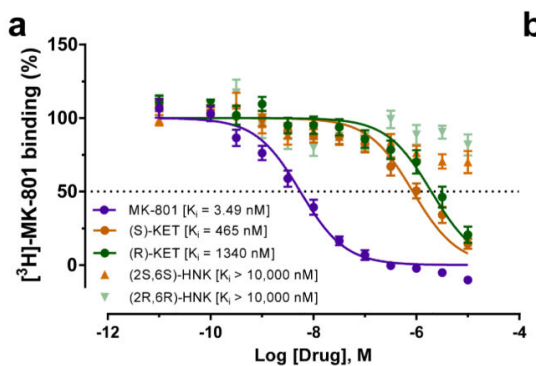

b
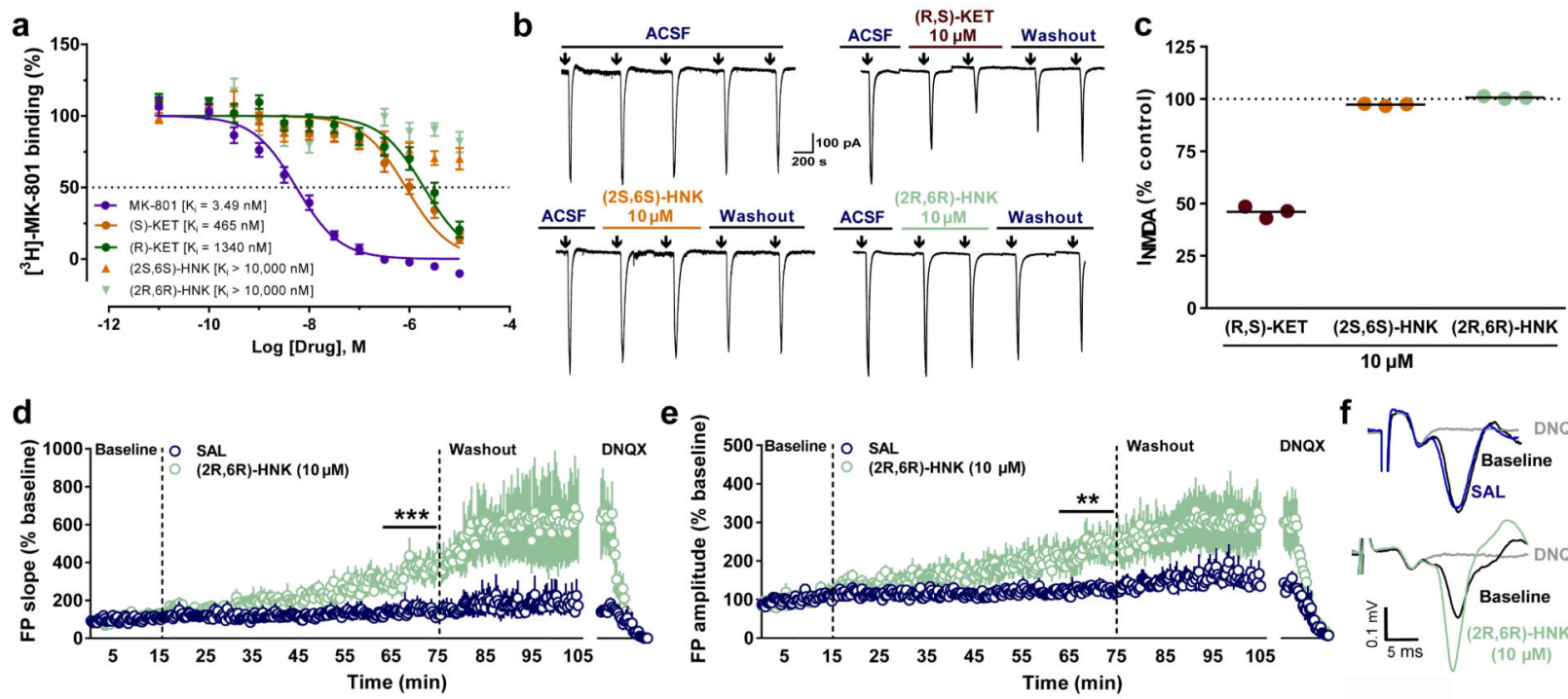

g

$* * *$
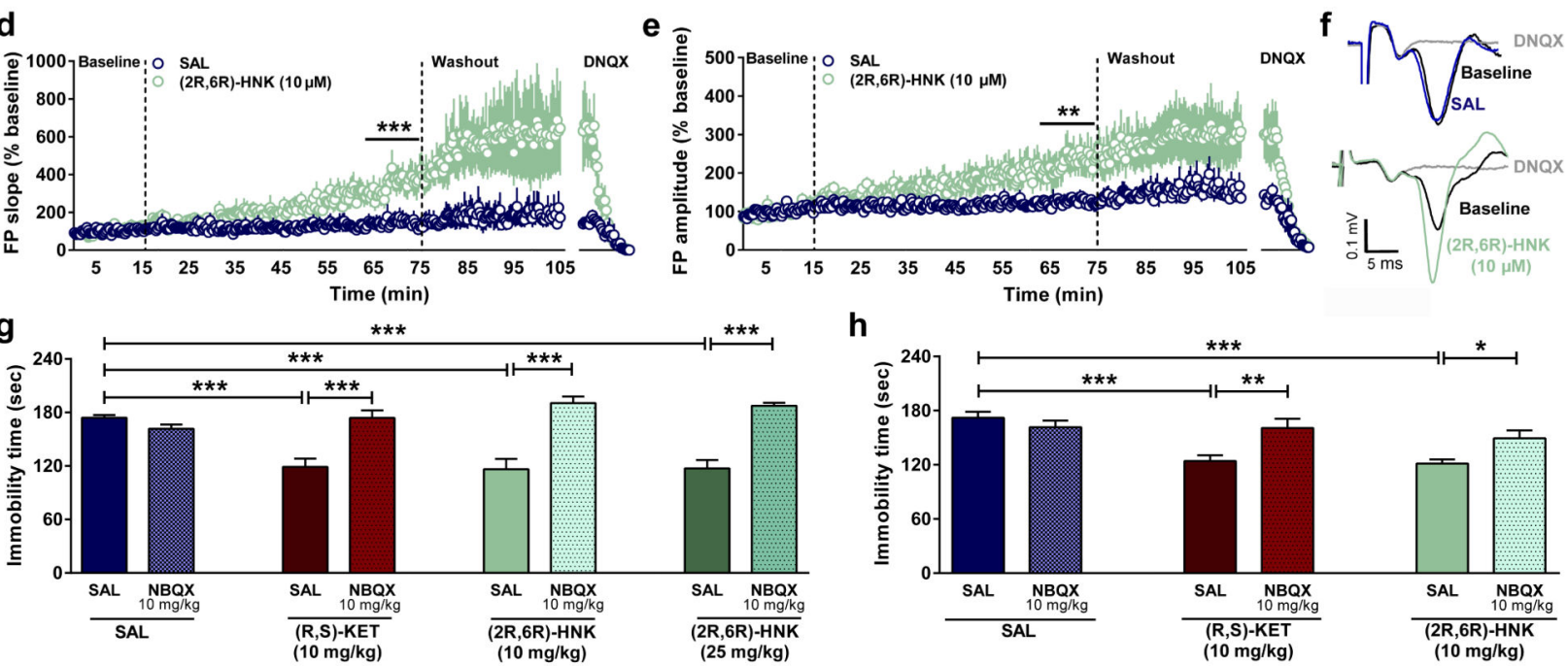

h
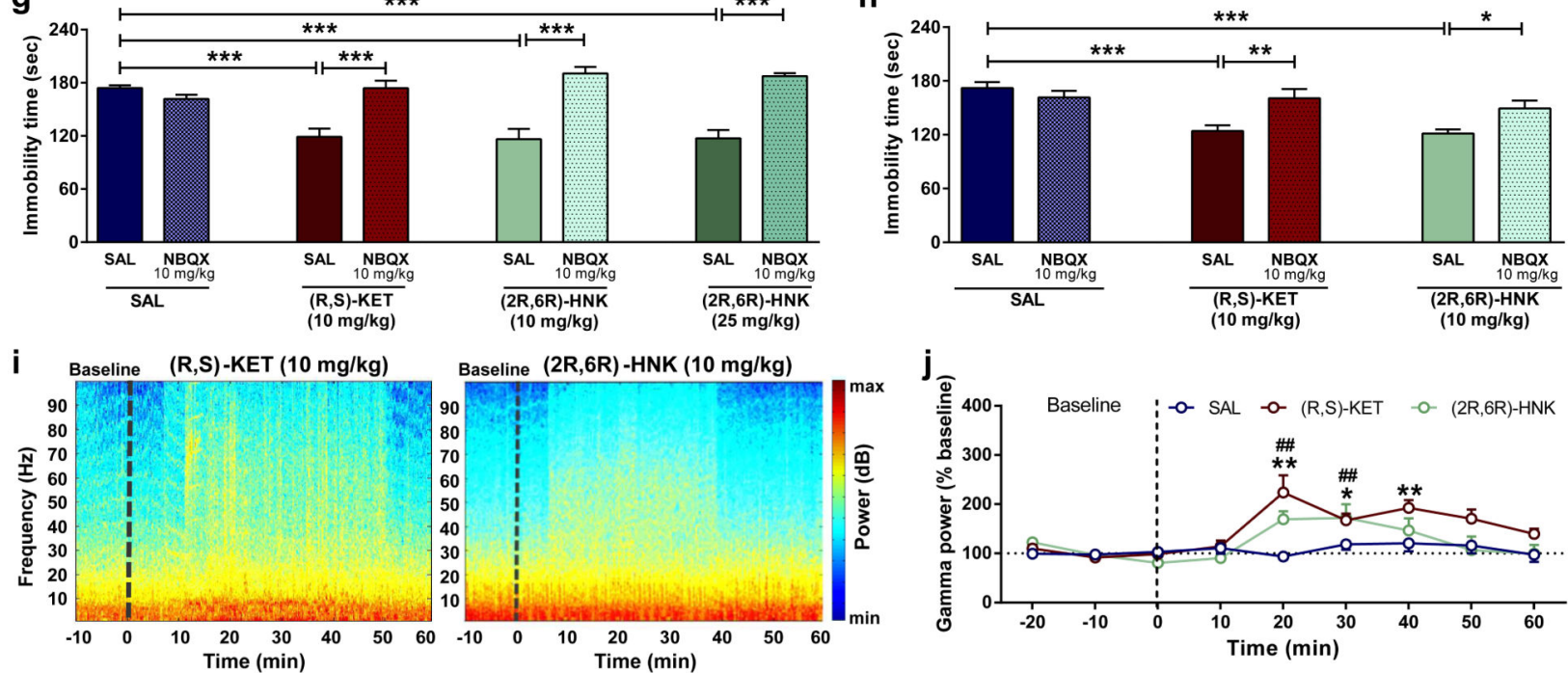

Figure 3. Activation of AMPA receptors is necessary for the acute antidepressant effects of (2R, 6R)-HNK

a, (2R,6R)-hydroxynorketamine (HNK) does not displace [ $\left.{ }^{3} \mathrm{H}\right]-\mathrm{MK}-801$ binding. (b-c), (R,S)-ketamine inhibited, but (2S,6S)-HNK and (2R,6R)-HNK did not inhibit b, currents evoked by application of NMDA to stratum radiatum interneurons in rat hippocampal slices, quantified as c, percent inhibition ( $\downarrow$ : 30-sec pulse). (d-e), Normalised d, fEPSP slope and e, amplitude from stimulation of the Schaffer collateral pathway in rat hippocampal slices. $\mathbf{f}$, Representative field-potential traces in the same hippocampal slice before (baseline) and 60 min after application of (2R,6R)-HNK. (g-h), Pre-treatment with the AMPA receptor inhibitor NBQX 10 minutes prior to (R,S)-ketamine (KET) and (2R,6R)-HNK prevented their antidepressant-like actions in the $\mathbf{g}, 1$-hour or $\mathbf{h}, 24$-hours forced-swim test. $\mathbf{i}$, Representative EEG spectrograms for 10-min prior (baseline) and 1-hour after administration of (R,S)-ketamine or (2R,6R)-HNK (indicated by a dashed line). $\mathbf{j}$, Normalised gamma power changes following administration of (R,S)-KET, (2R,6R)-HNK, 
or vehicle. Data are means \pm S.E.M. $* p<0.05, * * p<0.01, * * * p<0.001$ (statistical analyses and $n$ numbers see Supplementary Information Table 1). 


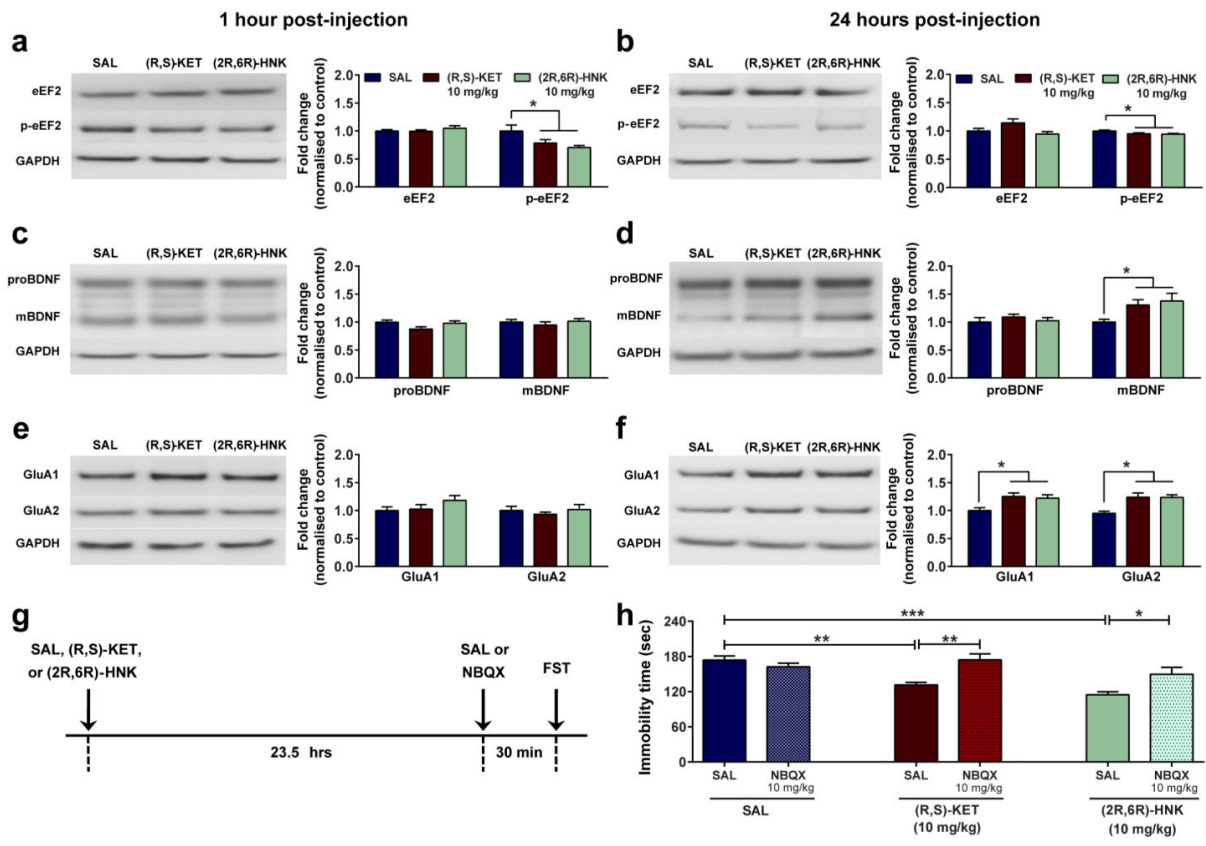

Figure 4. Activation of AMPA receptors is required for the sustained antidepressant effects of (2R,6R)-HNK

(a-h) Protein and protein phosphorylation levels from hippocampal synaptoneurosomes. (a,b) A single administration of (R,S)-ketamine (KET) or (2R,6R)-hydroxynorketamine (HNK) decreased phosphorylation of eEF2, a, 1 hour and b, 24 hours post-injection. (c,d), While administration of (2R,6R)-HNK or (R,S)-KET did not alter the levels of proBDNF or mBDNF c, 1 hour post-injection, it increased mBDNF levels. d, 24 hours post-treatment. (e,f), (R,S)-KET and (2R,6R)-HNK did not change levels of GluA1 and GluA2 at e, 1-hour but did f increase at 24-hours post-treatment. (g,h), Administration of the AMPA receptor inhibitor NBQX, 30 min prior to the 24-hour forced-swim test prevented the antidepressant effects of both (R,S)-KET and (2R,6R)-HNK administered 23.5 hours prior to NBQX. Data are means \pm S.E.M. Images cropped; see Supplementary Fig. 1 for complete blot images. $* p<0.05, * *, p<0.01, * * * p<0.001$ (statistical analyses and $n$ numbers see Supplementary Information Table 1). Abbreviations: : eEF2, eukaryotic translation elongation factor 2; FST, forced-swim test; GAPDH, Glyceraldehyde 3-phosphate dehydrogenase; mBDNF, mature brain-derived neurotrophic factor; NBQX, 2,3-dihydroxy-6-nitro-7-sulfamoylbenzo[f]quinoxaline-2,3-dione; proBDNF, pro-brain-derived neurotrophic factor; SAL, saline. 
a
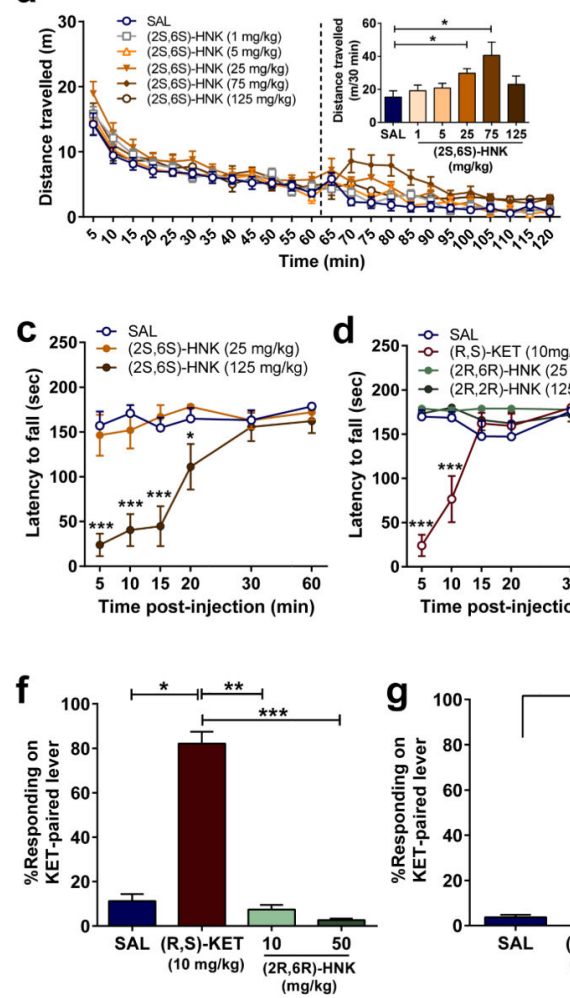

b
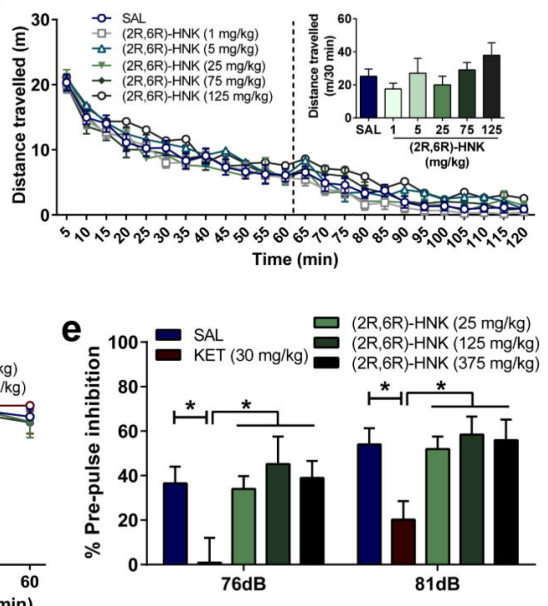

h $\quad{ }^{100} 1$ - 0 (R,S)-KET
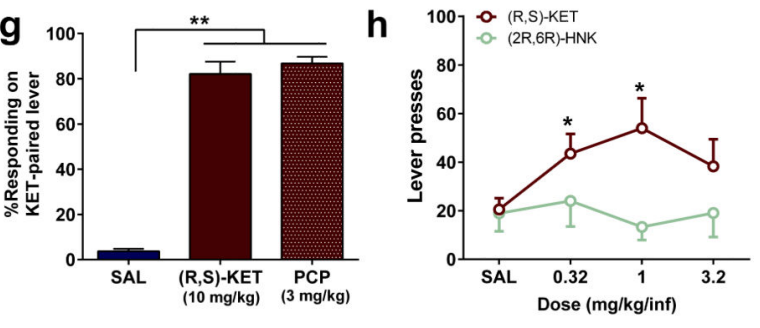

Figure 5. (2R,6R)-HNK lacks ketamine-related side effects

(a,b) After recording baseline activity for 1 hour, mice received drug (dashed line) and locomotor activity was monitored for 1 hour. a, Administration of $(2 \mathrm{~S}, 6 \mathrm{~S})$ -

hydroxynorketamine (HNK) dose-dependently changed locomotor activity, while administration of $\mathbf{b},(2 \mathrm{R}, 6 \mathrm{R})-\mathrm{HNK}$ did not. $\mathbf{c},(2 \mathrm{~S}, 6 \mathrm{~S})-\mathrm{HNK}$, but not $\mathbf{d},(2 \mathrm{R}, 6 \mathrm{R})-\mathrm{HNK}$, induced motor in-coordination in the rotarod. Unlike (R,S)-KET, (2R,6R)-HNK administration did not induce e, pre-pulse inhibition deficits, (f,g), (R,S)-KET-associated discriminative stimulus, or h, self-administration. Data are means \pm S.E.M. ${ }^{*} p<0.05, * *$, $p<0.01, * * * p<0.001$, KET vs saline (SAL); for panel c, * $(\mathrm{R}, \mathrm{S})-\mathrm{KET},{ }^{*}(2 \mathrm{~S}, 6 \mathrm{~S})-\mathrm{HNK}$ (statistical analyses and $n$ numbers see Supplementary Information Table 1). 